ESAIM: M2AN 47 (2013) 57-81

DOI: $10.1051 / \mathrm{m} 2 \mathrm{an} / 2012019$
ESAIM: Mathematical Modelling and Numerical Analysis

www.esaim-m2an.org

\title{
CONSISTENCY, ACCURACY AND ENTROPY BEHAVIOUR OF REMESHED PARTICLE METHODS
}

\author{
Lisl Weynans ${ }^{1,2,3}$ AND Adrien Magni ${ }^{4}$
}

\begin{abstract}
In this paper we analyze the consistency, the accuracy and some entropy properties of particle methods with remeshing in the case of a scalar one-dimensional conservation law. As in [G.-H. Cottet and L. Weynans, C. R. Acad. Sci. Paris, Ser. I 343 (2006) 51-56] we re-write particle methods with remeshing in the finite-difference formalism. This allows us to prove the consistency of these methods, and accuracy properties related to the accuracy of interpolation kernels. Cottet and Magni devised recently in [G.-H. Cottet and A. Magni, C. R. Acad. Sci. Paris, Ser. I 347 (2009) 1367-1372] and [A. Magni and G.-H. Cottet, J. Comput. Phys. 231 (2012) 152-172] TVD remeshing schemes for particle methods. We extend these results to the nonlinear case with arbitrary velocity sign. We present numerical results obtained with these new TVD particle methods for the Euler equations in the case of the Sod shock tube. Then we prove that with these new TVD remeshing schemes the particle methods converge toward the entropy solution of the scalar conservation law.
\end{abstract}

Mathematics Subject Classification. 65M12, 65M75.

Received October 12, 2011. Revised April 5, 2012.

Published online July 31, 2012.

\section{INTRODUCTION}

Particle methods are Lagrangian techniques that have been designed for advection-dominated physical problems. In this class of methods, the fluid is discretized on small masses concentrated on points: the particles, which are moved in a Lagrangian way. The classical particle methods used in fluid dynamics are Smoothed Particle Hydrodynamics (SPH) $[2,11,25]$ introduced by Monaghan and Particle-In-Cell (PIC) methods [9, 12]. If nothing is done, the distribution of particles becomes less and less uniform as time goes on, because they accumulate naturally in certain zones, for instance near strong gradients, and rarefy elsewhere. This phenomenon can lead to a loss of accuracy. A common remedy to this problem consists in periodically creating new particles uniformly distributed by an interpolation of the values of the existing particles, what is usually called remeshing the particles. The remeshing step creates new particles in a conservative way, by distributing the

Keywords and phrases. Particle methods with remeshing, interpolation kernels, consistency, truncation error, entropy inequalities, total variation, limiters, convergence.

1 Univ. Bordeaux, IMB, UMR 5251, 33400 Talence, France. lisl.weynans@math.u-bordeaux1.fr

2 CNRS, IMB, UMR 5251, 33400 Talence, France.

3 INRIA, 33400 Talence, France.

4 Laboratoire Jean Kuntzmann, Université Joseph Fourier, 51 rue des Mathématiques, 38041 Grenoble Cedex 9, France.

adrien.magni@imag.fr 
quantities carried by the particles at the nodes of an underlying grid. The frequency at which the interpolation must be performed depends on the simulated flow, but it is often chosen to remesh particles at each time step. This choice allows to solve the non-convective part of the considered equations (pressure gradient, diffusion for instance) with variables located on a grid, thus more easily as if they were irregularly distributed. The ability of particle methods with remeshing to simulate satisfactorily fluid dynamics has been studied and validated in the past: $[5,8,16,26,27]$ for instance. More recent works include $[4,15,29]$.

In [7] remeshed particle methods were rewritten as finite-difference methods and analyzed in this formalism. For example the particle scheme corresponding to a second-order interpolation kernel named $\Lambda_{2}$ was found to be equivalent to the Lax-Wendroff scheme in the linear case, whereas in the nonlinear case it provided a new finite-difference scheme. In this paper we keep on focusing on the finite-difference analysis of remeshed particles methods, and study their properties of consistency, accuracy and convergence toward entropy solution on a one-dimensional nonlinear scalar transport equation in an infinite domain:

$$
\partial_{t} u+\partial_{x}(g(u) u)=0, \quad t \geq 0,-\infty<x<+\infty .
$$

A convergence proof in $L_{\mathrm{loc}}^{p}$ has been established in [1] for a weighted particle method belonging to SPH methods which are purely Lagrangian methods. In $[17,18]$ are studied the convergence in $L^{2}$ and $L^{1}$ of renormalized SPH methods. To our knowledge, such an analysis for remeshed particle methods has not been performed yet. Moreover, thanks to the flux limiting, we will deal here with higher degree interpolation kernels than in the latter references, where the kernels were assumed to be positive, and thus performed only linear interpolation. In Section 2 we briefly recall the principles of remeshed particle methods, and the interpolation kernels that are classically used to perform remeshing. In Section 3 we present how a finite-difference scheme can be derived from the particle method with remeshing. Then we study the consistency and accuracy of remeshed particle methods under a CFL condition. Cottet and Magni introduced recently in [6, 20,21] a way to perform flux limiting on particles schemes and make them TVD. In Section 4 we present how TVD remeshing schemes can be built for nonlinear conservation laws with arbitrary sign of the particle velocity, and a numerical application to the Euler equation. In Section 5 we study the convergence of these TVD particle methods toward the entropy solution. This study is motivated by a numerical observation: contrary to some finite-difference schemes, as the Lax-Wendroff scheme for instance, the particle methods with remeshing seem to converge toward the entropy solution of the Burgers equation, as it is noticed in [6]. Using techniques inspired by [22], we prove that the new TVD remeshing schemes converge in the $L_{\text {loc }}^{1}$ norm toward the entropy solution of the scalar conservation law.

\section{Particle methods With Remeshing}

\subsection{Particle discretization}

Here we present how a particle method with remeshing can be introduced to solve the model transport equation (1.1). If we express this equation using the Lagrangian derivative associated to the material velocity $g(u)$, that is,

$$
\frac{\mathrm{d} u}{\mathrm{~d} t}=\partial_{t} u+g(u) \partial_{x} u
$$

we get

$$
\frac{\mathrm{d} u}{\mathrm{~d} t}+\partial_{x}(g(u)) u=0
$$

Let $V(t)$ be a material volume element moving at the flow velocity. By applying the Reynolds theorem, we get

$$
\frac{\mathrm{d}}{\mathrm{d} t}\left(\int_{V(t)} u \mathrm{~d} V\right)=\int_{V(t)} \frac{\mathrm{d} u}{\mathrm{~d} t} \mathrm{~d} V+\int_{V(t)} u \partial_{x}(g(u)) \mathrm{d} V .
$$


Thus,

$$
\frac{\mathrm{d}}{\mathrm{d} t}\left(\int_{V(t)} u \mathrm{~d} V\right)=0 .
$$

Following this property, the particle discretization consists in cutting the fluid into small masses concentrated on points: the particles. Each particle $j$ has a location $x_{j}$, carries the constant quantity $m_{j}=V_{j} u_{j}$, with $V_{j}$ the volume of the particle, and moves at velocity $g\left(u_{j}\right)$. The variables carried by the particles satisfy the equations

$$
\begin{aligned}
\frac{\mathrm{d} m_{j}}{\mathrm{~d} t} & =0, \\
\frac{\mathrm{d} x_{j}}{\mathrm{~d} t} & =g\left(u_{j}\right) .
\end{aligned}
$$

To solve this system with the particle method, one has to move particles during one time step and then interpolate them on the nodes of the underlying uniform grid. All particles are initially located on the nodes of a uniform grid, with space step $\Delta x$. The volumes of particles are equal to the cell volumes. We note $x_{j}^{n}$ the location of particle $j$ at time $n \Delta t, u_{j}^{n}$ the value of $u$ carried by the particle, and $\tilde{g}(u)_{i}^{n}$ the velocity at which the particle is moved. $\tilde{g}(u)_{i}^{n}$ may be equal to $g\left(u_{i}^{n}\right)$ but not necessarily. An example will be given later. It may also be a function of several variables providing a consistent approximation of $g(u)$ :

$$
\tilde{g}(u)_{i}^{n}=F\left(u_{j-m}, \ldots, u_{j+m}\right) .
$$

For consistency reasons that will appear in the proof of the consistency in Section 3.2 we impose that $F(u, \ldots, u)=g(u)$.

\subsection{Interpolation kernels}

In this section we shortly review interpolation kernels commonly used for the class of particle methods considered here. More details can be found in [5]. We only present one-dimensional interpolation kernels, because very often interpolation kernels in higher dimensions are devised by tensorial products of one-dimensional interpolation formulas.

Let a distribution of particles be indexed with $q$, located in $x_{q}$, carrying quantities $m_{q}$. For instance, in the case of Euler equations for gas dynamics, $m_{q}$ can be the mass, the momentum or the total energy of particle $q$. For incompressible flows, in the case of Vortex-In-Cell methods, $m_{q}$ is the vorticity. Let $W$ be an interpolation kernel. The remeshing process creates new particles at the nodes of a uniform underlying grid, with space step $\Delta x$. The new quantities $\tilde{m}_{i}$ at grid points $\tilde{x}_{i}$ are computed from the former values with the formula

$$
\tilde{m}_{i}=\sum_{q} m_{q} W\left(\frac{\tilde{x}_{i}-x_{q}}{\Delta x}\right) .
$$

The usual interpolation kernels are symmetrical, so as not to favour one direction compared to the others. With a Fourier analysis one can prove that the order of the interpolation is equal to the number of momenta preserved by the new particle distribution, i.e.,

$$
\begin{aligned}
& \sum_{i} \tilde{m}_{i}=\sum_{q} m_{q}, \\
& \sum_{i} \tilde{m}_{i}\left(x-\tilde{x}_{i}\right)=\sum_{q} m_{q}\left(x-x_{q}\right), \\
& \sum_{i} \tilde{m}_{i}\left(x-\tilde{x}_{i}\right)^{2}=\sum_{q} m_{q}\left(x-x_{q}\right)^{2} . \\
& \ldots
\end{aligned}
$$


This property is true up to the order $M-1$ if the kernel $W$ satisfies (see Sect. 7.2 in [5])

$$
\sum_{i} W\left(\frac{x-\tilde{x}_{i}}{\Delta x}\right) \tilde{x}_{i}^{a}=x^{a} \text { for all } x \text { and } 0 \leq a \leq M-1 .
$$

A family of interpolation kernels can be built by imposing the conservation of a given number of momenta $M$ with a minimal number of grid points. $\Lambda_{1}$ is the first kernel built with this principle, with $M=2$. It preserves the first two momenta of the particle distribution.

$$
\Lambda_{1}(x)= \begin{cases}1-|x| & \text { if }|x| \leq 1 \\ 0 & \text { if }|x|>1\end{cases}
$$

This kernel is in practise very diffusive and is not very used by itself. The next interpolation kernel, preserving the first three momenta of the particle distribution, is:

$$
\Lambda_{2}(x)= \begin{cases}1-x^{2} & \text { if }|x| \leq 1 / 2 \\ (1-|x|)(2-|x|) / 2 & \text { if } 1 / 2<|x| \leq 3 / 2 \\ 0 & \text { if }|x| \geq 1.5\end{cases}
$$

Although successfully used in the past, this kernel has the drawback of being very dispersive. This is partially explained by the fact that it is not continuous: a small error on the location of a particle can thus result in a large error on the values of the particles created by the interpolation step. The following interpolation kernels of this family, $\Lambda_{3}$ and $\Lambda_{4}$, need respectively four and five grid points and preserve one or two additionnal momenta.

Other interpolation kernels can be built by successive convolutions of $\Lambda_{1}$. They are of increasing regularity, but only preserve the first two momenta thus it is only possible to perform linear interpolation with them. The kernel $M_{3}$, which is $C^{1}$, is traditionnally referred to as the TSC (triangular-shaped cloud) interpolation function in particle-in-cell methods.

$$
M_{3}(x)= \begin{cases}1 / 2(x+3 / 2)^{2}-3 / 2(x+1 / 2)^{2} & \text { if }|x| \leq 1 / 2 \\ 1 / 2(-|x|+3 / 2)^{2} & \text { if } 1 / 2 \leq|x| \leq 3 / 2 \\ 0 & \text { if }|x|>3 / 2\end{cases}
$$

$M_{4}$ and $M_{5}$ are the following kernels of this family, respectively $C^{2}$ and $C^{3}$. Monaghan [24] devised the well known $M_{4}^{\prime}$ kernel with a Richardson extrapolation from a linear combination of $M_{4}$ and its derivative.

$$
M_{4}^{\prime}(x)= \begin{cases}1-5 x^{2} / 2+3|x|^{3} / 2 & \text { if }|x| \leq 1 \\ (2-|x|)^{2}(1-|x|) / 2 & \text { if } 1<|x| \leq 2 \\ 0 & \text { if }|x| \geq 2\end{cases}
$$

This kernel is $C^{1}$ and preserves the first three momenta.

\section{Consistency And ACCURACy of particle methods With Remeshing}

\subsection{Results}

Following the notations introduced in Section 2.1, let $u_{j}^{n}$ denote the value carried by the particle initially located on grid point $j$, at time $n \Delta t$. It is possible to express $u_{j}^{n+1}$ as a function of the $u_{i}^{n}$.

Proposition 3.1. If we note $\tilde{g}(u)_{i}^{n}$ the velocity used to move the particle $i$ between the times $n \Delta t$ and $(n+1) \Delta t$, and $\Lambda$ the interpolation kernel used for the remeshing step, we can express the particle method with remeshing as a finite-difference scheme:

$$
u_{j}^{n+1}=\sum_{i} u_{i}^{n} \Lambda\left(j-i-\frac{\Delta t \tilde{g}(u)_{i}^{n}}{\Delta x}\right) .
$$


Remark 3.2. In the latter formula, we use the symbol $\sum_{i}$ without specifying to which interval belong the $i$ indices. Because the interpolation kernel considered here have all compact supports, but of various sizes, this notation avoids us to specify the support of the considered interpolation kernel.

Proof. The location of the particle $i$ after being moved is

$$
\tilde{x}_{i}=x_{i}+\Delta t \tilde{g}(u)_{i}^{n} .
$$

The particles are remeshed on the same uniform grid as the one on which they were initially defined. Thus the locations of the new particles are again the nodes of the grid, and their volumes are all equal to $\Delta x$. The new particle distribution is computed from the old one with the interpolation formula

$$
m_{j}^{n+1}=\sum_{i} m_{i}^{n} \Lambda\left(\frac{x_{j}-\tilde{x}_{i}}{\Delta x}\right) .
$$

We can re-write the latter equation as

$$
u_{j}^{n+1} V_{j}^{n+1}=\sum_{i} u_{i}^{n} V_{i}^{n} \Lambda\left(\frac{x_{j}-x_{i}-\Delta t \tilde{g}(u)_{i}^{n}}{\Delta x}\right) .
$$

As $V_{j}^{n+1}=V_{i}^{n}=\Delta x$ this equation simplifies to

$$
u_{j}^{n+1}=\sum_{i} u_{i}^{n} \Lambda\left(\frac{x_{j}-x_{i}-\Delta t \tilde{g}(u)_{i}^{n}}{\Delta x}\right),
$$

$x_{i}$ and $x_{j}$ being the nodes of a uniform grid, with space step $\Delta x$, we have

$$
\frac{x_{j}-x_{i}}{\Delta x}=j-i \text {. }
$$

We finally get

$$
u_{j}^{n+1}=\sum_{i} u_{i}^{n} \Lambda\left(j-i-\frac{\Delta t}{\Delta x} \tilde{g}(u)_{i}^{n}\right) .
$$

We recognize the form of a finite-difference or finite-volume scheme. For example, if we develop this formula with the kernel $\Lambda_{2}$ in the linear case $g(u)=a>0$, with the CFL condition $\left|a \frac{\Delta t}{\Delta x}\right|<\frac{1}{2}$, we get the Lax-Wendroff scheme. Monaghan [23] had already noticed a similarity between particle methods and finite-difference methods. In [10], Wee et Ghoniem used an analysis similar to ours to build modified interpolation kernels taking into account diffusion terms. The formula (3.1) is difficult to interpret by itself, because the weights associated to the values $u_{i}^{n}$ are expressed with the kernel $\Lambda$. But it is possible to obtain several properties of the particle scheme only with the knowledge of the number of momenta preserved by the interpolation kernel $\Lambda$. The first one adresses the consistency of particle methods:

Proposition 3.3. Let $\Lambda$ be an interpolation kernel piecewise polynomial of degree $N$ with compact support, which preserves at least the first two momenta. The scheme (3.1) can be written in a conservative form consistent with equation (1.1).

To solve a flow where shocks may appear, it is crucial that the numerical scheme can be written in conservative form consistent with the equation to solve. In hyperbolic problems this property ensures the scheme to satisfy the discrete Rankine-Hugoniot conditions across discontinuities. Thus, if the scheme is converging, it converges necessarily toward a weak solution of the considered equation. At the contrary, a non-conservative scheme used to solve conservation laws will have a problematic convergence, as addressed in $[3,14]$. 
One can distinguish two kinds of interpolation kernels: kernels whose support size is an even integer (for example kernels $\Lambda_{1}, \Lambda_{3}, M_{4}$ and $M_{4}^{\prime}$ ), and kernels whose support size is odd (for example $\Lambda_{2}$ and $M_{3}$ ). For the sake of brevity, in the following we will call them respectively kernels with even or odd support. The finitedifference stencil provided by kernels with even support varies with the sign of the velocity of the particles. For this reason these kernels suffer from problems of consistency in the finite-difference sense (visible through an analysis of the truncation error) if the velocity of the particles changes sign, as it is proved in [20,21]. On the contrary, kernels with odd support like $\Lambda_{2}$ are defined on intervals $[k-1 / 2, k+1 / 2]$, and the formula used to interpolate a particle on a grid point does not depend on the velocity of the particle. Under a CFL condition $\left|g\left(u_{j}\right) \frac{\Delta t}{\Delta x}\right|<\frac{1}{2}$, these kernels do not suffer from consistency problems. In the following we will therefore focus our study on kernels with odd support. The second property that we will prove adresses the accuracy of particle schemes:

Proposition 3.4. Let $\Lambda$ be an interpolation kernel with odd support, piecewise polynomial of degree $N$, which preserves the first $M$ momenta, and $u$ a solution of equation (1.1). For a given $n \geq 0$, we denote $u_{j}^{n}=u(j \Delta x, n \Delta t)$. If we suppose that the functions $u$ et $\tilde{g}$ are at least of class $C^{M-1}$, and the CFL condition $\left|g\left(u_{j}\right) \frac{\Delta t}{\Delta x}\right|<\frac{1}{2}$ is satisfied, then $u_{j}^{n+1}$ defined by (3.1) satisfies

$$
u_{j}^{n+1}=\sum_{i=0}^{M-1} \frac{\Delta t^{i}}{i !}(-1)^{i} \frac{\partial^{i}\left(u \tilde{g}^{i}\right)}{\partial x^{i}}(j \Delta x, n \Delta t)+O\left(\Delta x^{M}\right) .
$$

This property is also satisfied by kernels with even support, if the sign of the velocity of the particles is constant. In this case actually, the stencil used to remesh the particles does not change, and the reasoning is the same as for kernels with odd support. The Proofs of Propositions 3.3 et 3.4 are detailed in Section 3.2.

The formula (3.2) allows us to evaluate the accuracy of the particle method with respect to time and space. We assume that $\Delta x$ and $\Delta t$ are proportional. If we take $\tilde{g}(u)=g(u)$, the truncation error $E_{j}^{n+1}$ is

$$
\begin{aligned}
E_{j}^{n+1}= & \frac{u(j \Delta x,(n+1) \Delta t)-u_{j}^{n+1}}{\Delta t} \\
= & \frac{u(j \Delta x,(n+1) \Delta t-u(j \Delta x, n \Delta t))}{\mathrm{d} t}+\partial_{x}(g(u) u)(j \Delta x, n \Delta t) \\
& -\frac{\Delta t}{2} \partial_{x x}\left(g(u)^{2} u\right)(j \Delta x, n \Delta t)+O\left(\Delta x^{2}\right) \\
= & \partial_{t} u(j \Delta x, n \Delta t)+\frac{\Delta t}{2} \partial_{t t} u(j \Delta x, n \Delta t)+\partial_{x}(g(u) u)(j \Delta x, n \Delta t) \\
& -\frac{\Delta t}{2} \partial_{x x}\left(g(u)^{2} u\right)(j \Delta x, n \Delta t)+O\left(\Delta x^{2}\right)+O\left(\Delta t^{2}\right) \\
= & O(\Delta t) .
\end{aligned}
$$

The scheme is thus first-order accurate even if the interpolation kernel preserves a higher number of momenta, because of the non-zero term $\partial_{t t} u-\partial_{x x}\left(g(u)^{2} u\right)$. Actually, the choice $\tilde{g}(u)=g(u)$ consists in moving the particle during the time step $\Delta t$ with its velocity evaluated at the beginning of the time step

$$
\tilde{x}_{j}=x_{j}+\Delta \operatorname{tg}\left(u_{j}\right) .
$$

It is an explicit first-order Euler scheme, and consequently the particle moving is also order one. Therefore, in order to increase the scheme accuracy, the velocity of particles during time step $\Delta t$ needs to be evaluated with a better accuracy. In [7] was introduced a new Runge-Kutta 2 advancing scheme for the particles, allowing to recover in the nonlinear case a second-order accuracy. The idea is to use the velocity of the particle at the middle of the time step, at $t+\frac{\Delta t}{2}$. Because this value is not exactly known, it is replaced by a second-order 
approximation: $g\left(u+\frac{\Delta t}{2} \frac{\mathrm{d} u}{\mathrm{~d} t}\right)$. Thus we move the particles at velocity

$$
\tilde{g}(u)=g\left(u+\frac{\Delta t}{2} \frac{\mathrm{d} u}{\mathrm{~d} t}\right)
$$

that is,

$$
\tilde{x}_{j}=x_{j}+\Delta t g\left(u+\frac{\Delta t}{2} \frac{\mathrm{d} u}{\mathrm{~d} t}\right)_{x=x_{j}} .
$$

Proposition 3.5. For smooth enough solutions, the particle scheme (3.1) computed with the corrected velocity (3.3) is second-order accurate if $\Lambda$ preserves at least the first three momenta.

Proof. The scheme truncation error becomes

$$
\begin{aligned}
E_{j}^{n+1}= & \frac{u(j \Delta x,(n+1) \Delta t)-u_{j}^{n+1}}{\Delta t} \\
= & \partial_{t} u(j \Delta x, n \Delta t)+\frac{\Delta t}{2} \partial_{t t} u(j \Delta x, n \Delta t)+\partial_{x}\left(g\left(u+\frac{\Delta t}{2} \frac{\mathrm{d} u}{\mathrm{~d} t}\right) u\right)(j \Delta x, n \Delta t) \\
& -\frac{\Delta t}{2} \partial_{x x}\left(g\left(u+\frac{\Delta t}{2} \frac{\mathrm{d} u}{\mathrm{~d} t}\right)^{2} u\right)(j \Delta x, n \Delta t)+O\left(\Delta x^{2}\right) \\
= & \partial_{t} u(j \Delta x, n \Delta t)+\frac{\Delta t}{2} \partial_{t t} u(j \Delta x, n \Delta t)+\partial_{x}(g(u) u)(j \Delta x, n \Delta t) \\
& +\frac{\Delta t}{2} \partial_{x}\left(\frac{\mathrm{d} u}{\mathrm{~d} t} u g^{\prime}(u)\right)(j \Delta x, n \Delta t)-\frac{\Delta t}{2} \partial_{x x}\left(g(u)^{2} u\right)(j \Delta x, n \Delta t)+O\left(\Delta x^{2}\right) .
\end{aligned}
$$

We want to prove that the sum $\partial_{t t} u+\partial_{x}\left(\frac{\mathrm{d} u}{\mathrm{~d} t} u g^{\prime}(u)\right)-\partial_{x x}\left(g(u)^{2} u\right)$ vanishes. Indeed,

$$
\begin{aligned}
\partial_{t t} u & =-\partial_{t x}(g(u) u)=-\partial_{x}\left(g^{\prime}(u) u \partial_{t} u+g(u) \partial_{t} u\right)=\partial_{x}\left[\left(g^{\prime}(u) u+g(u)\right) \partial_{x}(g(u) u)\right], \\
\frac{\mathrm{d} u}{\mathrm{~d} t} & =-\partial_{x}(g(u)),
\end{aligned}
$$

and we check that

$$
\partial_{x}\left[\left(g^{\prime}(u) u+g(u)\right) \partial_{x}(g(u) u)\right]-\partial_{x}\left(\partial_{x}(g(u)) u g^{\prime}(u)\right)-\partial_{x x}\left(g(u)^{2} u\right)=0
$$

Thus we obtain

$$
E_{j}^{n+1}=O\left(\Delta x^{2}\right) .
$$

\subsection{Proof of Propositions 3.3 and 3.4}

We present in this subsection the Proofs of Propositions 3.3 and 3.4 in the case of a kernel with odd support. These proofs are based on the fact that interpolation kernels preserve a certain number of momenta. We begin with the proof of a lemma that will be used in the following.

Lemma 3.6. Let $\Lambda$ be an interpolation kernel with odd support, piecewise polynomial and preserving the first $M$ momenta as in (2.3). For all $m$ and $i$ such that $0 \leq i \leq M-1$, we have

$$
\sum_{k} k^{i} \Lambda^{(m)}(k)=(-1)^{i} i ! \delta_{i}^{m} .
$$


Proof. As $\Lambda$ preserves the first $M$ momenta, we rewrite the formula (2.3) with $\tilde{x}_{k}=k$ and $\Delta x=1$.

$$
\sum_{k} k^{i} \Lambda(k-x)=x^{i} \quad 0 \leq i \leq M-1 .
$$

Because $\Lambda$ has an odd support, it is differentiable on each interval $[k-1 / 2, k+1 / 2]$, and we deduce from the latter formula that

$$
\begin{gathered}
\text { if } i=0 \text { and } m>0 \sum_{k} k^{i} \Lambda^{(m)}(k-x)=0, \\
\quad \text { if } i=m=0 \sum_{k} k^{i} \Lambda^{(m)}(k-x)=\sum_{k} \Lambda(k-x)=1, \\
\text { if } i \neq 0 \text { and } 0 \leq m \leq i \sum_{k} k^{i}(-1)^{m} \Lambda^{(m)}(k-x)=i(i-1) \ldots(i-m+1) x^{i-m}, \\
\text { if } i \neq 0 \text { and } m>i \sum_{k} k^{i}(-1)^{m} \Lambda^{(m)}(k-x)=0 .
\end{gathered}
$$

Thus, for $x=0$ we have

$$
\begin{aligned}
\text { if } i=0 \text { and } m>0 & \sum k^{i} \Lambda^{(m)}(k)=0, \\
\text { if } i=m=0 & \sum_{k} k^{i} \Lambda(k)=1, \\
\text { if } i \neq 0 \text { and } m \neq i & \sum_{k} k^{i} \Lambda^{(m)}(k)=0, \\
\text { if } i \neq 0 \text { and } m=i & \sum_{k} k^{i} \Lambda^{(m)}(k)=(-1)^{i} i ! .
\end{aligned}
$$

Proof of Proposition 3.3. Consistency of the scheme:

Let $u$ be a smooth solution of equation $\partial_{t} u+\partial_{x}(g(u) u)=0$ for a given initial condition. Let us denote

$$
u_{j}^{n}=u(j \Delta x, n \Delta t) \text { for all } n \geq 0 \text { and } j .
$$

We consider formula (3.1) written in a slightly different form, namely,

$$
u_{j}^{n+1}=\sum_{k} u_{j+k}^{n} \Lambda\left(k+\frac{\Delta t}{\Delta x} \tilde{g}(u)_{j+k}^{n}\right) .
$$

For the sake of clarity we note $\tilde{g}_{j+k}=\tilde{g}(u)_{j+k}^{n}, u_{j}=u_{j}^{n}$ and $\lambda=\frac{\Delta t}{\Delta x}$. Formula (3.5) becomes

$$
u_{j}^{n+1}=\sum_{k} u_{j+k} \Lambda\left(k+\lambda \tilde{g}_{j+k}\right) .
$$

Let $N$ be the degree of $\Lambda$. The kernel $\Lambda$ being differentiable on each interval $[k-1 / 2, k+1 / 2]$, and because of the CFL condition, we can develop each term in a Taylor expansion

$$
\Lambda\left(k+\lambda \tilde{g}_{j+k}\right)=\sum_{i=0}^{N} \frac{\Lambda^{(i)}(k)}{i !}\left(\lambda \tilde{g}_{j+k}\right)^{i} .
$$

We deduce from the latter that

$$
u_{j}^{n+1}=\sum_{k} \sum_{i=0}^{N} \frac{\lambda^{i}}{i !} \Lambda^{(i)}(k) u_{j+k} \tilde{g}_{j+k}^{i} .
$$


As we have assumed that the support of $\Lambda$ is compact, it can be included in $[-d, d]$, with $d$ an integer, and equation (3.6) can be re-written as

$$
u_{j}^{n+1}=\sum_{k=-d, k \neq 0}^{d}\left[\sum_{i=0}^{N} u_{j+k} \frac{\lambda^{i}}{i !} \Lambda^{(i)}(k) \tilde{g}_{j+k}^{i}\right]+\left[\sum_{i=0}^{N} u_{j} \frac{\lambda^{i}}{i !} \Lambda^{(i)}(0) \tilde{g}_{j}^{i}\right] .
$$

As $\sum_{k} \Lambda(k+x)=1$, we have

$$
\Lambda^{(i)}(0)=\delta_{0}^{i}-\sum_{k \neq 0} \Lambda^{(i)}(k)
$$

Thus,

$$
\begin{gathered}
u_{j}^{n+1}=u_{j}+\sum_{k=-d, k \neq 0}^{d} \sum_{i=0}^{N} \frac{\lambda^{i}}{i !} \Lambda^{(i)}(k)\left[u_{j+k} \tilde{g}_{j+k}^{i}-u_{j} \tilde{g}_{j}^{i}\right], \\
u_{j}^{n+1}=u_{j}+\sum_{k=1}^{d} \sum_{i=0}^{N} \frac{\lambda^{i}}{i !}\left[\Lambda^{(i)}(k)\left[u_{j+k} \tilde{g}_{j+k}^{i}-u_{j} \tilde{g}_{j}^{i}\right]+\Lambda^{(i)}(-k)\left[u_{j-k} \tilde{g}_{j-k}^{i}-u_{j} \tilde{g}_{j}^{i}\right]\right] .
\end{gathered}
$$

We notice that

$$
\begin{aligned}
& u_{j+k} \tilde{g}_{j+k}^{i}-u_{j} \tilde{g}_{j}^{i}=\sum_{a=1}^{k} u_{j+a} \tilde{g}_{j+a}^{i}-\sum_{a=0}^{k-1} u_{j+a} \tilde{g}_{j+a}^{i}, \\
& u_{j-k} \tilde{g}_{j-k}^{i}-u_{j} \tilde{g}_{j}^{i}=-\sum_{a=-k+1}^{0} u_{j+a} \tilde{g}_{j+a}^{i}+\sum_{a=-k}^{-1} u_{j+a} \tilde{g}_{j+a}^{i} .
\end{aligned}
$$

Thus,

$$
\begin{aligned}
u_{j}^{n+1}= & u_{j}+\sum_{k=1}^{d} \sum_{i=0}^{N} \frac{\lambda^{i}}{i !}\left[\Lambda^{(i)}(k)\left[\sum_{a=1}^{k} u_{j+a} \tilde{g}_{j+a}^{i}-\sum_{a=0}^{k-1} u_{j+a} \tilde{g}_{j+a}^{i}\right]\right. \\
& \left.+\Lambda^{(i)}(-k)\left[-\sum_{a=-k+1}^{0} u_{j+a} \tilde{g}_{j+a}^{i}+\sum_{a=-k}^{-1} u_{j+a} \tilde{g}_{j+a}^{i}\right]\right] .
\end{aligned}
$$

We can then write

$$
u_{j}^{n+1}=u_{j}-\lambda\left[G\left(u_{j+d}, \ldots, u_{j-d+1}\right)-G\left(u_{j+d-1}, \ldots, u_{j-d}\right)\right]
$$

with

$$
G\left(u_{j+d}, \ldots, u_{j-d+1}\right)=-\sum_{k=1}^{d} \sum_{i=0}^{N} \frac{\lambda^{i-1}}{i !}\left[\Lambda^{(i)}(k) \sum_{a=1}^{k} u_{j+a} \tilde{g}_{j+a}^{i}-\Lambda^{(i)}(-k) \sum_{a=-k+1}^{0} u_{j+a} \tilde{g}_{j+a}^{i}\right] .
$$

Consequently the scheme (3.1) can be written in conservative form. Moreover,

$$
\begin{aligned}
G(u, \ldots, u) & =-\sum_{k=1}^{d} \sum_{i=0}^{N} \frac{\lambda^{i-1}}{i !}\left[\Lambda^{(i)}(k) \sum_{a=1}^{k} u g(u)^{i}-\Lambda^{(i)}(-k) \sum_{a=-k+1}^{0} u g(u)^{i}\right] \\
& =-\sum_{k=1}^{d} \sum_{i=0}^{N} u g(u)^{i} \frac{\lambda^{i-1}}{i !}\left[k \Lambda^{(i)}(k)-k \Lambda^{(i)}(-k)\right] .
\end{aligned}
$$


According to Lemma 3.6, we have then

$$
G(u, \ldots, u)=u g(u) .
$$

The scheme (3.1) is therefore consistent with equation (1.1).

Proof of Proposition 3.4. Accuracy of the scheme:

We start again from formula (3.5)

$$
u_{j}^{n+1}=\sum_{k} u_{j+k} \Lambda\left(k+\lambda \tilde{g}_{j+k}\right) .
$$

The kernel $\Lambda$ being differentiable on each interval $[k-1 / 2, k+1 / 2]$, we have

$$
\Lambda\left(k+\lambda \tilde{g}_{j+k}\right)=\sum_{i=0}^{N} \frac{\Lambda^{(i)}(k)}{i !}\left(\lambda \tilde{g}_{j+k}\right)^{i}
$$

Thus,

$$
u_{j}^{n+1}=\sum_{k} \sum_{i=0}^{N} \frac{\lambda^{i}}{i !} \Lambda^{(i)}(k) u_{j+k} \tilde{g}_{j+k}^{i} .
$$

We define $\left(f_{i}\right)_{j+k}=u_{j+k} \tilde{g}_{j+k}^{i}$ and develop these terms in a Taylor series

$$
\left(f_{i}\right)_{j+k}=\sum_{a=0}^{M-1} \frac{\partial^{a}\left(f_{i}\right)}{\partial x^{a}}\left(x_{j}\right) \frac{k^{a} \Delta x^{a}}{a !}+O\left(\Delta x^{M}\right) .
$$

Thus,

$$
u_{j}^{n+1}=\sum_{k} \sum_{i=0}^{N} \frac{\lambda^{i}}{i !} \sum_{a=0}^{M-1} \frac{k^{a} \Delta x^{a}}{a !} \Lambda^{(i)}(k) \frac{\partial^{a}\left(f_{i}\right)}{\partial x^{a}}\left(x_{j}\right)+O\left(\Delta x^{M}\right),
$$

and we swap the order of summation to yield

$$
u_{j}^{n+1}=\sum_{i=0}^{N} \frac{\lambda^{i}}{i !} \sum_{a=0}^{M-1} \frac{\Delta x^{a}}{a !} \frac{\partial^{a}\left(f_{i}\right)}{\partial x^{a}}\left(x_{j}\right) \sum_{k} k^{a} \Lambda^{(i)}(k)+O\left(\Delta x^{M}\right) .
$$

With the results of Lemma 3.6, we simplify the latter expression as

$$
\begin{aligned}
u_{j}^{n+1} & =\sum_{i=0}^{M-1} \frac{\lambda^{i}}{i !} \frac{\Delta x^{i}}{i !}\left[\frac{\partial^{i}\left(f_{i}\right)}{\partial x^{i}}\left(x_{j}\right)(-1)^{i} i !\right]+O\left(\Delta x^{M}\right) \\
& =\sum_{i=0}^{M-1} \frac{\lambda^{i}}{i !}(-1)^{i} \Delta x^{i} \frac{\partial^{i}\left(u \tilde{g}(u)^{i}\right)}{\partial x^{i}}\left(x_{j}\right)+O\left(\Delta x^{M}\right) .
\end{aligned}
$$

\section{TVD REMESHING FORMULAS}

Recently Cottet and Magni derived in [6,20,21] TVD remeshing formulas for particle methods. This subsection gives the principles to construct TVD remeshing formulas based on the $\Lambda_{2}$ kernel in the nonlinear case and for CFL conditions less than 1/2, avoiding consistency problems evocated in [21]. Examples are given for Burgers and Euler equations. 


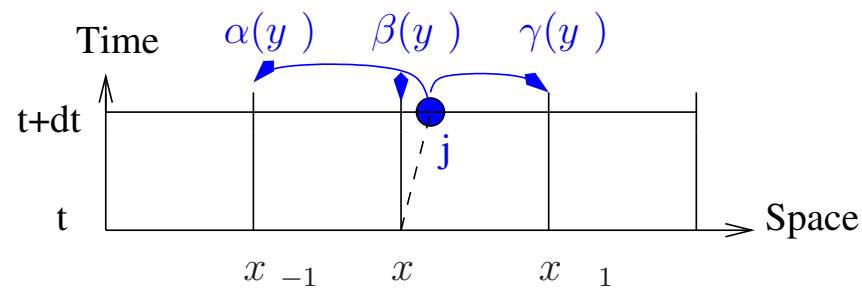

Figure $1 . \Lambda_{2}$ remeshing formulas, $0<\lambda \tilde{g}<1 / 2$.

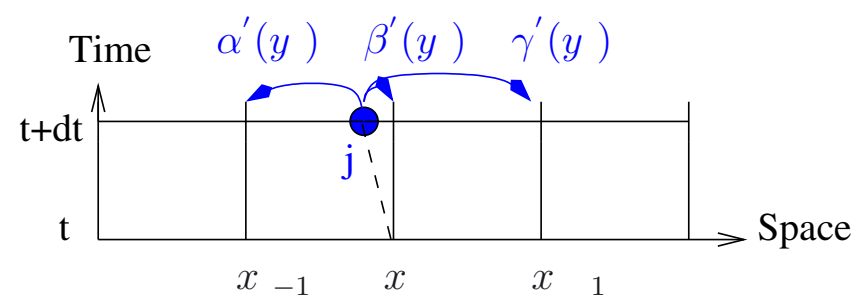

FiguRE 2. $\Lambda_{2}$ remeshing formulas, $-1 / 2<\lambda \tilde{g}<0$.

\subsection{Principle of TVD remeshing schemes}

Let the velocity be positive $(0<\lambda \tilde{g}<1 / 2)$. The remeshing of a particle with the $\Lambda_{2}$ kernel is equivalent to assigning the weights

$$
\left\{\begin{array}{l}
\alpha\left(y_{j}\right)=\alpha_{j}=y_{j}\left(y_{j}-1\right) / 2 \\
\beta\left(y_{j}\right)=\beta_{j}=1-y_{j}^{2} \\
\gamma\left(y_{j}\right)=\gamma_{j}=y_{j}\left(y_{j}+1\right) / 2
\end{array}\right.
$$

to the amount carried by the particle and redistribute it on the neighbouring grid points. This is depicted in Figure 1, where $y_{j}=\lambda \tilde{g_{j}}$ is the relative distance between the remeshed particle $j$ and the grid point at the left. The scheme resulting from the $\Lambda_{2}$ remeshing (4.1) at the grid point $x_{j}$ and the time $t^{n}=n \Delta t$ is

$$
u_{j}^{n+1}=\sum_{k} u_{j+k}^{n} \Lambda_{2}\left(k+\lambda \tilde{g}_{j+k}\right)=\gamma_{j-1} u_{j-1}^{n}+\beta_{j} u_{j}^{n}+\alpha_{j+1} u_{j+1}^{n} .
$$

If now the velocity is negative $(-1 / 2<\lambda \tilde{g}<0)$ as shown in Figure 2 , the weights are

$$
\left\{\begin{array}{l}
\alpha^{\prime}\left(y_{j}\right)=\alpha\left(y_{j}-1\right) \\
\beta^{\prime}\left(y_{j}\right)=\beta\left(y_{j}-1\right) \\
\gamma^{\prime}\left(y_{j}\right)=\gamma\left(y_{j}-1\right)
\end{array}\right.
$$

and the scheme is

$$
u_{j}^{n+1}=\gamma_{j-1}^{\prime} u_{j-1}^{n}+\beta_{j}^{\prime} u_{j}^{n}+\alpha_{j+1}^{\prime} u_{j+1}^{n} .
$$

Since $y_{j}=\lambda \tilde{g_{j}}+1,(4.2)$ is equivalent to (4.4) and a unique scheme is given at the point $x_{j}$ independently of the velocity sign. The order of accuracy is two, and the drawback of this scheme is to be not TVD, therefore creating spurious oscillations. In order to have a TVD scheme, it is possible to introduce some numerical diffusion in (4.2) by adding a parameter $\sigma$ to the remeshing formulas (4.1)-(4.3). This new formula (4.5) is called $\bar{M}_{3}$, in reference to the $M_{3}$ formula obtained when $\sigma=1 / 8$ and used in traditional Particle In Cell methods. The interpolation kernel $\bar{M}_{3}$ preserves the first two momenta and thus provides order one accuracy. The value of 
$\sigma$ will be evaluated in Section 4.2 to ensure the TVD property of the $\bar{M}_{3}$ scheme.

$$
\begin{cases}\alpha^{M 3}\left(y_{j}\right)=y_{j}\left(y_{j}-1\right) / 2+\sigma, & \alpha^{M 3^{\prime}}\left(y_{j}\right)=\alpha^{M 3}\left(y_{j}-1\right) \\ \beta^{M 3}\left(y_{j}\right)=1-y_{j}^{2}-2 \sigma, & \beta^{M 3^{\prime}}\left(y_{j}\right)=\beta^{M 3}\left(y_{j}-1\right) \\ \gamma^{M 3}\left(y_{j}\right)=y_{j}\left(y_{j}+1\right) / 2+\sigma, & \gamma^{M 3^{\prime}}\left(y_{j}\right)=\gamma^{M 3}\left(y_{j}-1\right) .\end{cases}
$$

A TVD remeshing formula of order two in smooth regions can be built introducing a limiter $\phi$ to combine the TVD formula $\bar{M}_{3}$ (obtained when $\phi=0$ ) and the second order $\Lambda_{2}$ formula $(\phi=1)$. The weights $(4.1)$ and $(4.3)$ must be replaced respectively by (4.6) if $0<\lambda \tilde{g}<1 / 2$ and (4.7) if $-1 / 2<\lambda \tilde{g}<0$.

$$
\begin{gathered}
\left\{\begin{array}{l}
\alpha^{T V D}\left(y_{j}, \phi\right)=y_{j}\left(y_{j}-1\right) / 2+\sigma\left(1-\phi_{j-1 / 2}\right) \\
\beta^{T V D}\left(y_{j}, \phi\right)=1-y_{j}^{2}-\sigma\left(1-\phi_{j-1 / 2}\right)-\sigma\left(1-\phi_{j+1 / 2}\right) \\
\gamma^{T V D}\left(y_{j}, \phi\right)=y_{j}\left(y_{j}+1\right) / 2+\sigma\left(1-\phi_{j+1 / 2}\right),
\end{array}\right. \\
\left\{\begin{array}{l}
\alpha^{T V D^{\prime}}\left(y_{j}, \bar{\phi}\right)=\alpha^{T V D}\left(y_{j}-1, \bar{\phi}\right) \\
\beta^{T V D^{\prime}}\left(y_{j}, \bar{\phi}\right)=\beta^{T V D}\left(y_{j}-1, \bar{\phi}\right) \\
\gamma^{T V D^{\prime}}\left(y_{j}, \bar{\phi}\right)=\gamma^{T V D}\left(y_{j}-1, \bar{\phi}\right) .
\end{array}\right.
\end{gathered}
$$

The limiters $\phi_{j \pm 1 / 2}=\phi\left(r_{j \pm 1 / 2}\right)$ with $r_{j+1 / 2}=\left(u_{j}-u_{j-1}\right) /\left(u_{j+1}-u_{j}\right), r_{j-1 / 2}=\left(u_{j-1}-u_{j-2}\right) /\left(u_{j}-u_{j-1}\right)$ and $\bar{\phi}_{j \pm 1 / 2}=\phi\left(\bar{r}_{j \pm 1 / 2}\right)$ with $\bar{r}_{j+1 / 2}=\left(u_{j+2}-u_{j+1}\right) /\left(u_{j+1}-u_{j}\right), \bar{r}_{j-1 / 2}=\left(u_{j+1}-u_{j}\right) /\left(u_{j}-u_{j-1}\right)$ will be calculated in Section 4.3 to ensure the TVD property of the scheme for all grid points $x_{j}$.

In the case of a velocity changing sign, we must take care about the limiters. $\phi(r) \neq \phi(\bar{r})$ so the scheme in some grid points is different of (4.2) and (4.4). To overcome this difficulty it is possible to replace formulas (4.6)(4.7) to remesh some specific particles. More precisely, if the particle $j-1$ has a positive velocity and $j$ has a negative velocity, this particle must be remeshed by the formulas

$$
\left\{\begin{array}{l}
\alpha_{j}^{T V D^{\prime}}=\left(y_{j}-1\right)\left(\left(y_{j}-1\right)-1\right) / 2+\sigma\left(1-\phi\left(\mathbf{r}_{\mathbf{j}-\mathbf{1} / \mathbf{2}}\right)\right) \\
\beta_{j}^{T V D^{\prime}}=1-\left(y_{j}-1\right)^{2}-\sigma\left(1-\phi\left(\mathbf{r}_{\mathbf{j}-\mathbf{1} / \mathbf{2}}\right)\right)-\sigma\left(1-\phi\left(\overline{\mathbf{r}}_{\mathbf{j}+\mathbf{1} / \mathbf{2}}\right)\right) \\
\gamma_{j}^{T V D^{\prime}}=\left(y_{j}-1\right)\left(\left(y_{j}-1\right)+1\right) / 2+\sigma\left(1-\phi\left(\overline{\mathbf{r}}_{\mathbf{j}+\mathbf{1} / \mathbf{2}}\right)\right) .
\end{array}\right.
$$

In the same way, if $j-1$ has a negative velocity and $j$ is a particle with positive velocity, it must be remeshed with

$$
\left\{\begin{array}{l}
\alpha_{j}^{T V D}=y_{j}\left(y_{j}-1\right) / 2+\sigma\left(1-\phi\left(\overline{\mathbf{r}}_{\mathbf{j}-\mathbf{1} / \mathbf{2}}\right)\right) \\
\beta_{j}^{T V D}=1-y_{j}^{2}-\sigma\left(1-\phi\left(\overline{\mathbf{r}}_{\mathbf{j}-\mathbf{1} / \mathbf{2}}\right)\right)-\sigma\left(1-\phi\left(\mathbf{r}_{\mathbf{j}+\mathbf{1} / \mathbf{2}}\right)\right) \\
\gamma_{j}^{T V D}=y_{j}\left(y_{j}+1\right) / 2+\sigma\left(1-\phi\left(\mathbf{r}_{\mathbf{j}+\mathbf{1} / \mathbf{2}}\right)\right) .
\end{array}\right.
$$

Remark 4.1. The TVD remeshing formulas (4.6)-(4.9) are still consistent with an order one accuracy at least, and conservative since the sum of the weights used to remesh any particle is one.

\subsection{The TVD scheme $\bar{M}_{3}$}

We give the proof of the TVD property of the $\bar{M}_{3}$ scheme discussing on the value of the parameter $\sigma$. This parameter can be viewed as an artificial viscosity since when $\sigma=0$ the $\bar{M}_{3}$ scheme reduced to the $\Lambda_{2}$ scheme, and to the $M_{3}$ scheme when $\sigma=1 / 8$. Let $f$ and $h$ be the two following functions

$$
\begin{aligned}
& f(u, g(u))=\lambda g u(\lambda g+1) \\
& h(u, g(u))=\lambda g u(\lambda g-1) .
\end{aligned}
$$

Let us define $\Delta f_{j+1 / 2}=f_{j+1}-f_{j}, \Delta f_{j-1 / 2}=f_{j}-f_{j-1}$, and the same notation holds replacing $f$ by $h$ or $u$. If the particles are remeshed with the formulas (4.6)-(4.7), the scheme at the grid point $x_{j}$ is

$$
u_{j}^{n+1}=u_{j}^{n}+C_{j+1 / 2}\left(u_{j+1}^{n}-u_{j}^{n}\right)-D_{j-1 / 2}\left(u_{j}^{n}-u_{j-1}^{n}\right),
$$


with

$$
\left\{\begin{array}{l}
C_{j+1 / 2}=\sigma+\frac{1}{2} \frac{\Delta h_{j+1 / 2}}{\Delta u_{j+1 / 2}} \\
D_{j-1 / 2}=\sigma+\frac{1}{2} \frac{\Delta f_{j-1 / 2}}{\Delta u_{j-1 / 2}}+\sigma\left(\frac{\phi_{j+1 / 2}}{r_{j+1 / 2}}-\phi_{j-1 / 2}\right),
\end{array}\right.
$$

and following the Taylor-Lagrange formula, there exists $\tilde{u} \in] u_{j-1}, u_{j}[$ and $\tilde{\tilde{u}} \in] u_{j-1}, u_{j}[$ such as

$$
\left\{\begin{array}{l}
C_{j-1 / 2}=\sigma+\frac{1}{2} h^{\prime}(\tilde{\tilde{u}}) \\
D_{j-1 / 2}=\sigma+\frac{1}{2} f^{\prime}(\tilde{u})+\sigma\left(\frac{\phi_{j+1 / 2}}{r_{j+1 / 2}}-\phi_{j-1 / 2}\right) .
\end{array}\right.
$$

The scheme $\bar{M}_{3}$ is reached doing $\phi=0$ in (4.13). Following the Harten theorem [13], the scheme (4.11) will be TVD if

$$
\left\{\begin{array}{l}
C_{j-1 / 2} \geq 0 \\
0 \leq D_{j-1 / 2} \leq 1-C_{j-1 / 2} .
\end{array}\right.
$$

Or if for all $u$

$$
\left\{\begin{array}{l}
\sigma+\frac{1}{2} h^{\prime}(u) \geq 0 \\
\sigma+\frac{1}{2} f^{\prime}(u) \geq 0 \\
2 \sigma+\frac{1}{2} f^{\prime}(u)+\frac{1}{2} h^{\prime}(u) \leq 1,
\end{array}\right.
$$

which is written again

$$
\left\{\begin{array}{l}
\sigma+\frac{1}{2} \lambda g(\lambda g-1)+\frac{1}{2} g^{\prime}(u) \lambda u(2 \lambda g-1) \geq 0 \\
\sigma+\frac{1}{2} \lambda g(\lambda g+1)+\frac{1}{2} g^{\prime}(u) \lambda u(2 \lambda g+1) \geq 0 \\
2 \sigma+\lambda^{2} g^{2}+2 g^{\prime}(u) \lambda^{2} g u \leq 1
\end{array}\right.
$$

Assuming

$$
\left\{\begin{array}{l}
|\lambda g| \leq C \\
\left|\lambda g^{\prime}\right| \leq C^{\prime}
\end{array}\right.
$$

we have

$$
\begin{aligned}
\frac{1}{2}\left|f^{\prime}\right| & =\frac{1}{2}\left|h^{\prime}\right| \\
& \leq \frac{1}{2}|\lambda g|^{2}+\frac{1}{2}|\lambda g|+\frac{1}{2}|u|\left|\lambda g^{\prime}\right|+|\lambda g|\left|\lambda g^{\prime}\right||u| \\
& \leq \frac{1}{2} C(C+1)+\frac{1}{2} C^{\prime} \max |u|(1+2 C) \\
& \leq \sigma
\end{aligned}
$$

if $C$ and $C^{\prime} \max |u|$ are small enough. So,

$$
\left\{\begin{array}{l}
\frac{1}{2}\left|f^{\prime}\right| \leq \sigma \\
\frac{1}{2}\left|h^{\prime}\right| \leq \sigma
\end{array}\right.
$$

and choosing $\sigma=1 / 4$,

$$
\left\{\begin{array}{l}
\frac{1}{2}\left(\left|f^{\prime}\right|+\left|h^{\prime}\right|\right) \leq 2 \sigma=1 / 2 \\
\frac{1}{2}\left(\left|f^{\prime}\right|+\left|h^{\prime}\right|\right) \leq 1-2 \sigma=1 / 2
\end{array}\right.
$$

the scheme $\bar{M}_{3}$ is TVD. This proof holds in the general case, but requires some constraint on the velocity field and its derivative: (4.17) with $C$ and $C^{\prime} \max |u|$ small enough. When the velocity field is given, an optimal value of $\sigma$ can be determined with the CFL condition corresponding. We study the case of the Burgers and the Euler equations. 


\subsubsection{Burgers equation}

Let us consider the Burgers equation, i.e., $g(u)=u / 2$. Since $g^{\prime}(u)=1 / 2$ there exists two constants $C$ and $C^{\prime}$ satisfying (4.17) under a CFL condition. Knowing the velocity $g$, it is possible to find an optimal parameter $\sigma$ giving rise to the largest CFL condition under the TVD constraint for scheme $\bar{M}_{3}$.

Indeed, the conditions (4.16) becomes

$$
\left\{\begin{array}{l}
\sigma \geq \frac{1}{2} \lambda u-\frac{3}{8}(\lambda u)^{2} \\
\sigma \geq-\frac{1}{2} \lambda u-\frac{3}{8}(\lambda u)^{2} \\
\sigma \leq \frac{1}{2}-\frac{3}{8}(\lambda u)^{2} .
\end{array}\right.
$$

Setting $c=\lambda\left(\frac{u}{2}\right)$ and according to the two first inequalities of (4.21), $\sigma \geq 1 / 6$ if $|c| \geq \frac{1}{3}$. According to the last condition, CFL condition must be $\max |c| \leq \sqrt{2} / 3 \simeq 0.47$ to have $\sigma \leq 1 / 6$, and then we choose $\sigma=1 / 6$.

Remark 4.2. If $\sigma=1 / 4$ the CFL condition is reduced to $1 / \sqrt{6}=0.41$ and the $\bar{M}_{3}$ formula is more diffusive than with $\sigma=1 / 6$.

\subsubsection{Euler equations}

Let be $u_{1}=\rho, u_{2}=\rho u$ and $u_{3}=\rho E$. Then, the Euler equations are written

$$
\left\{\begin{array}{l}
\frac{\partial u_{1}}{\partial t}+\frac{\partial}{\partial x}\left(u_{1} \frac{u_{2}}{u_{1}}\right)=0 \\
\frac{\partial u_{2}}{\partial t}+\frac{\partial}{\partial x}\left(u_{2} \frac{u_{2}}{u_{1}}\right)=-\frac{\partial p}{\partial x} \\
\frac{\partial u_{3}}{\partial t}+\frac{\partial}{\partial x}\left(u_{3} \frac{u_{2}}{u_{1}}\right)=-\frac{\partial}{\partial x}\left(p \frac{u_{2}}{u_{1}}\right) \\
p=(\gamma-1)\left(u_{3}-\frac{1}{2} \frac{u_{2}^{2}}{u_{1}}\right) .
\end{array}\right.
$$

These equations are solved using a two-step splitting method. The presssure effects are solved in a Lagrange step and the convective part is solved in an advection step [29]. We are interested in this last step which consists to advect particles to the velocity $g\left(u_{1}, u_{2}, u_{3}\right)=u_{2} / u_{1}$ and remesh them on a grid with TVD formulas. In this paragraph, we find optimal parameters $\sigma$ to construct TVD formulas $\bar{M}_{3}$ allowing the largest CFL condition. The limiters used in the remeshing formulas (4.6)-(4.7) will be constructed in Section 4.3.

Since the optimal parameter depends on the amount $u$ carried by the particles, the calculation must be done for the three equations of the system (4.22). Let us consider the first equation. $u=u_{1}, g=u_{2} / u_{1}$ and $g^{\prime}=-u_{2} / u_{1}^{2}$, so with $c=\lambda u_{2} / u_{1}(4.16)$ becomes

$$
\left\{\begin{array}{l}
\sigma-\frac{1}{2} c^{2} \geq 0 \\
\sigma-\frac{1}{2} c^{2} \geq 0 \\
2 \sigma-c^{2} \leq 1 .
\end{array}\right.
$$

To ensure the consistency of the $\Lambda_{2}$ remeshing [21] $-1 / 2 \leq c \leq 1 / 2$, the CFL condition is $\max |c| \leq 1 / 2$ and $\sigma=1 / 8$.

In the same way, for the second equation of (4.22), $u=u_{2}, g=u_{2} / u_{1}, g^{\prime}=1 / u_{1}$ and (4.16) becomes

$$
\left\{\begin{array}{l}
\sigma+\frac{3}{2} c^{2}-c \geq 0 \\
\sigma+\frac{3}{2} c^{2}+c \geq 0 \\
2 \sigma+3 c^{2} \leq 1 .
\end{array}\right.
$$


As for Burgers equation, the optimal value for the parameter $\sigma$ is $1 / 6$ and the CFL condition is $\max |c| \leq \sqrt{2} / 3$.

Finally, for the energy equation, $u=u_{3}, g=u_{2} / u_{1}$ and $g^{\prime}=0$. So (4.16) becomes

$$
\left\{\begin{array}{l}
\sigma+\frac{1}{2} c^{2}-\frac{1}{2} c \geq 0 \\
\sigma+\frac{1}{2} c^{2}+\frac{1}{2} c \geq 0 \\
2 \sigma+c^{2} \leq 1,
\end{array}\right.
$$

and the optimal parameter is $\sigma=1 / 8$ allowing a CFL condition of $\sqrt{3} / 2$ which is reduced to $1 / 2$ for the consistency.

We want to use the same parameter $\sigma$ to remesh $\rho, \rho u$ or $\rho E$ with the $\bar{M}_{3}$ formula. So we chose the smallest value of sigma, i.e., $\sigma=1 / 6$, and the CFL condition is $\max |c| \leq \sqrt{2} / 3$.

\subsection{Calculation of the limiter $\phi(r)$}

Let us assume the velocity $g \geq 0$. The limiters are built from (4.11)-(4.13) using again the Harten theorem [13]. Assuming that the parameter $\sigma$ is chosen to have a $\bar{M}_{3}$ scheme TVD as explained in Section 4.2 , then the coefficient $C_{j-1 / 2}$ given in (4.12) is positive from the conditions (4.14). So the scheme (4.11) obtained using the $\Lambda_{2}$-TVD remeshing formulas (4.6)-(4.7) will be TVD if

$$
0 \leq D_{j-1 / 2} \leq 1-C_{j-1 / 2}
$$

This condition is written again

$$
0 \leq 1+\frac{1}{2 \sigma} f^{\prime}(u)-\phi_{j-1 / 2}+\frac{\phi_{j+1 / 2}}{r_{j+1 / 2}} \leq \frac{1}{\sigma}-1-\frac{1}{2 \sigma} h^{\prime}(u),
$$

which is verified if the limiters satisfy

$$
\left\{\begin{aligned}
0 \leq \phi_{j-1 / 2} & \leq 1+\frac{1}{2 \sigma} f^{\prime}(u) \\
& =1+\frac{1}{2 \sigma} \lambda^{2} g^{2}+\frac{1}{\sigma} \lambda^{2} g g^{\prime} u+\frac{1}{2 \sigma} \lambda g+\frac{1}{2 \sigma} \lambda g^{\prime} u \\
0 \leq \phi_{j+1 / 2} / r_{j+1 / 2} & \leq \frac{1}{\sigma}-1-\frac{1}{2 \sigma} h^{\prime}(u) \\
& =\frac{1}{\sigma}-2-\frac{1}{\sigma} \lambda^{2} g^{2}-\frac{2}{\sigma} \lambda^{2} g g^{\prime} u .
\end{aligned}\right.
$$

If the velocity $g \leq 0$, the coefficients $C_{j+1 / 2}$ and $D_{j-1 / 2}$ can be chosen in order to introduce the slope ratio $\bar{r}_{j-1 / 2}=\Delta u_{j+1 / 2} / \Delta u_{j-1 / 2}$ :

$$
\left\{\begin{array}{l}
C_{j+1 / 2}=\sigma+\frac{1}{2} \frac{\Delta h_{j+1 / 2}}{\Delta u_{j+1 / 2}}+\sigma\left(\frac{\phi_{j-1 / 2}}{\bar{r}_{j-1 / 2}}-\phi_{j+1 / 2}\right) \\
D_{j-1 / 2}=\sigma+\frac{1}{2} \frac{\Delta f_{j-1 / 2}}{\Delta u_{j-1 / 2}} .
\end{array}\right.
$$

The limiters are built by imposing $0 \leq C_{j+1 / 2} \leq 1-D_{j+1 / 2}$,

$$
\left\{\begin{array}{l}
0 \leq \phi_{j+1 / 2} \leq 1+\frac{1}{2 \sigma} \lambda^{2} g^{2}+\frac{1}{\sigma} \lambda^{2} g g^{\prime} u-\frac{1}{2 \sigma} \lambda g-\frac{1}{2 \sigma} \lambda g^{\prime} u \\
0 \leq \phi_{j-1 / 2} / \bar{r}_{j-1 / 2} \leq \frac{1}{\sigma}-2-\frac{1}{\sigma} \lambda^{2} g^{2}-\frac{2}{\sigma} \lambda^{2} g g^{\prime} u .
\end{array}\right.
$$

In the case of a velocity changing sign, particles are remeshed by the formulas (4.8) and (4.9). Then the scheme obtained on the grid point $x_{j}$ is

$$
\begin{aligned}
u_{j}^{n+1}= & u_{j}^{n}+\frac{1}{2}\left(h_{j+1}-h_{j}\right)+\sigma\left(u_{j+1}^{n}-u_{j}^{n}\right)-\frac{1}{2}\left(f_{j}-f_{j-1}\right)-\sigma\left(u_{j}^{n}-u_{j-1}^{n}\right) \\
& -\sigma\left[\phi\left(r_{j+1 / 2}\right)\left(u_{j+1}^{n}-u_{j}^{n}\right)-\phi\left(r_{j-1 / 2}\right)\left(u_{j}^{n}-u_{j-1}^{n}\right)\right] .
\end{aligned}
$$


This scheme will be TVD if

$$
\left\{\begin{array}{l}
\sigma+\frac{1}{2} f^{\prime}-\sigma \phi_{j-1 / 2}(r) \geq 0 \\
\sigma+\frac{1}{2} h^{\prime}-\sigma \phi_{j+1 / 2}(\bar{r}) \geq 0 \\
2 \sigma+\frac{1}{2}\left(f^{\prime}+h^{\prime}\right)-\sigma\left(\phi_{j+1 / 2}(\bar{r})+\phi_{j+1 / 2}(r)\right) \leq 1 .
\end{array}\right.
$$

The first equation is satisfied since (4.28) holds and the second also with (4.30). The last is also satisfied by virtue of inequality (4.26),

$$
2 \sigma+\frac{1}{2}\left(f^{\prime}+h^{\prime}\right)-\sigma \phi(\bar{r}) \leq 1-\sigma \frac{\phi(\bar{r})}{\bar{r}} \leq 1+\sigma \phi(r),
$$

since

$$
-\frac{\phi(\bar{r})}{\bar{r}} \leq 0 \leq \phi(r) .
$$

So, the scheme obtained in the case of a velocity changing sign is TVD if (4.28) and (4.30) hold.

\subsubsection{Examples}

As in Section 4.2, let us look at the case of the Burgers equation and the Euler equations. For the Burgers equation, $g(u)=u / 2, g^{\prime}(u)=1 / 2$, and $\sigma=1 / 6$, so always with $c=\lambda g$ equations (4.28) and (4.30) are written

$$
\left\{\begin{array}{l}
0 \leq \phi \leq 1 \leq 1+9 c^{2}+6|c| \\
0 \leq \phi \leq r\left(4-18 c^{2}\right)
\end{array}\right.
$$

Then the limiter is built in order to be as large as possible

$$
\phi(r)=\max \left\{0, \min \left[1+9 \max c^{2}+6 \max |c|,\left(4-18 \max c^{2}\right) r\right]\right\},
$$

or

$$
\phi(r)=\max \left\{0, \min \left[1,\left(4-18 \max c^{2}\right) r\right]\right\},
$$

and $r=\bar{r}$ if the velocity $g$ of the remeshed particle is negative.

For the Euler equations, $\sigma=1 / 6$. So, for the first equation (mass balance), (4.28)-(4.30) becomes

$$
\left\{\begin{array}{l}
0 \leq \phi \leq 1-\frac{1}{2 \sigma} c^{2}=1-3 c^{2} \\
0 \leq \phi \leq r\left(\frac{1}{\sigma}-2\right)=4 r \leq r\left(\frac{1}{\sigma}\left(1+c^{2}\right)-2\right) .
\end{array}\right.
$$

For the second Euler equation (momentum conservation), (4.28)-(4.30) becomes

$$
\left\{\begin{array}{l}
0 \leq \phi \leq 1 \leq 1+\frac{3}{2 \sigma} c^{2}+\frac{1}{\sigma} c=1+9 c^{2}+6 c, c \geq 0 \\
0 \leq \phi \leq 1 \leq 1+\frac{3}{2 \sigma} c^{2}-\frac{1}{\sigma} c=1+9 c^{2}-6 c, c \leq 0 \\
0 \leq \phi \leq r\left(\frac{1}{\sigma}-2\right)-\frac{3}{\sigma} c^{2}=r\left(4-18 c^{2}\right),
\end{array}\right.
$$

and for the last equation (energy conservation), (4.28)-(4.30) becomes

$$
\left\{\begin{array}{l}
0 \leq \phi \leq 1 \leq 1+\frac{1}{2 \sigma} c^{2}+\frac{1}{2 \sigma} c=1+3 c^{2}+3 c, c \geq 0 \\
0 \leq \phi \leq 1 \leq 1+\frac{1}{2 \sigma} c^{2}-\frac{1}{2 \sigma} c=1+3 c^{2}-3 c, c \leq 0 \\
0 \leq \phi \leq r\left(\frac{1}{\sigma}\left(1-c^{2}\right)-2\right)=r\left(4-6 c^{2}\right) .
\end{array}\right.
$$


We have chosen to remesh the variables $\rho, \rho u$ and $\rho E$ by the same formula. To do this, the same limiter must be used in (4.6)-(4.7), so it must satisfy in the same time inequalities (4.38)-(4.40). Setting

$$
r_{j+1 / 2}^{(1)}=\frac{\Delta \rho_{j-1 / 2}}{\Delta \rho_{j+1 / 2}}, \quad r_{j+1 / 2}^{(2)}=\frac{\Delta(\rho u)_{j-1 / 2}}{\Delta(\rho u)_{j+1 / 2}}, \quad r_{j+1 / 2}^{(3)}=\frac{\Delta(\rho E)_{j-1 / 2}}{\Delta(\rho E)_{j+1 / 2}},
$$

and

$$
\bar{r}_{j-1 / 2}^{(1)}=\frac{\Delta \rho_{j+1 / 2}}{\Delta \rho_{j-1 / 2}}, \quad \bar{r}_{j-1 / 2}^{(2)}=\frac{\Delta(\rho u)_{j+1 / 2}}{\Delta(\rho u)_{j-1 / 2}}, \quad \bar{r}_{j-1 / 2}^{(3)}=\frac{\Delta(\rho E)_{j+1 / 2}}{\Delta(\rho E)_{j-1 / 2}},
$$

the limiter is built like this:

$$
\phi\left(r^{(1)}, r^{(2)}, r^{(3)}\right)=\max \left\{0, \min \left[1-3 \max c^{2}, 4 r^{(1)},\left(4-18 \max c^{2}\right) r^{(2)},\left(4-6 \max c^{2}\right) r^{(3)}\right]\right\},
$$

replacing $r$ with $\bar{r}$ for negative velocities.

\subsection{Numerical validation: Sod shock tube}

In this section we present numerical results for the TVD particle methods applied to the one-dimensional Euler equations. We address the classical test case [28] of a shock tube initially made up two compartments, each containing a perfect gas $(\gamma=1.4)$. The initial conditions are

$$
\begin{aligned}
\boldsymbol{U}(x, 0)= & 0 \\
\rho(x, 0)= & 1 \text { if } 0 \leq x \leq 0.5 \\
& 0.125 \text { if } 0.5<x \leq 1 \\
P(x, 0)= & 2.5(\gamma-1) \text { if } 0 \leq x \leq 0.5 \\
& 0.25(\gamma-1) \text { if } 0.5<x \leq 1 .
\end{aligned}
$$

We solve the Euler equations using a splitting between the Lagrangian step, where are taken into account pressure effects, and the advection step, during which the particles are moved and remeshed. The Lagrangian step is solved with an approximate Riemann solver (acoustic approximation) with a limited MUSCL reconstruction. In Figure 3 are presented the results at $t=0.2$ for the density, velocity, pressure and thermal energy. The oscillations near the discontinuities observed in [29] that were created by classical interpolation kernels as $\Lambda_{2}$, $\Lambda_{3}$ or $M_{4}^{\prime}$ have disappeared.

\section{Convergence of TVD particle methods toward Entropy SOLUtion}

In this section we address the convergence of TVD particle schemes as defined in previous section toward the unique entropy solution of equation (1.1). In the remaining of the section we make the following assumptions:

- the initial condition $u_{0}$ of equation (1.1) has its total variation bounded: $T V\left(u_{0}\right)<+\infty$ and is bounded in $L^{\infty}$-norm;

- the function $g$ is of class $\mathrm{C}^{1}(\mathbb{R})$.

Let us give more details about how the numerical scheme that we study is defined. We denote $x_{j}=j \Delta x$, $x_{j+1 / 2}=(j+1 / 2) \Delta x$ and $t^{n}=n \Delta t$. We suppose that $\Delta t$ et $\Delta x$ are proportional to each other: $\frac{\Delta t}{\Delta x}=\lambda$ with $\lambda$ a constant. The sequence $\left(u_{j}^{n}\right)_{n \geq 0, j \in \mathbb{Z}}$ is defined by recurrence by equation (3.1) and the initial sequence $\left(u_{j}^{0}\right)_{n \geq 0, j \in \mathbb{Z}}$ is defined by

$$
u_{j}^{0}=\frac{1}{\Delta x} \int_{x_{j-1 / 2}}^{x_{j+1 / 2}} u_{0}(x) \mathrm{d} x, \text { for } j \in \mathbb{Z} .
$$



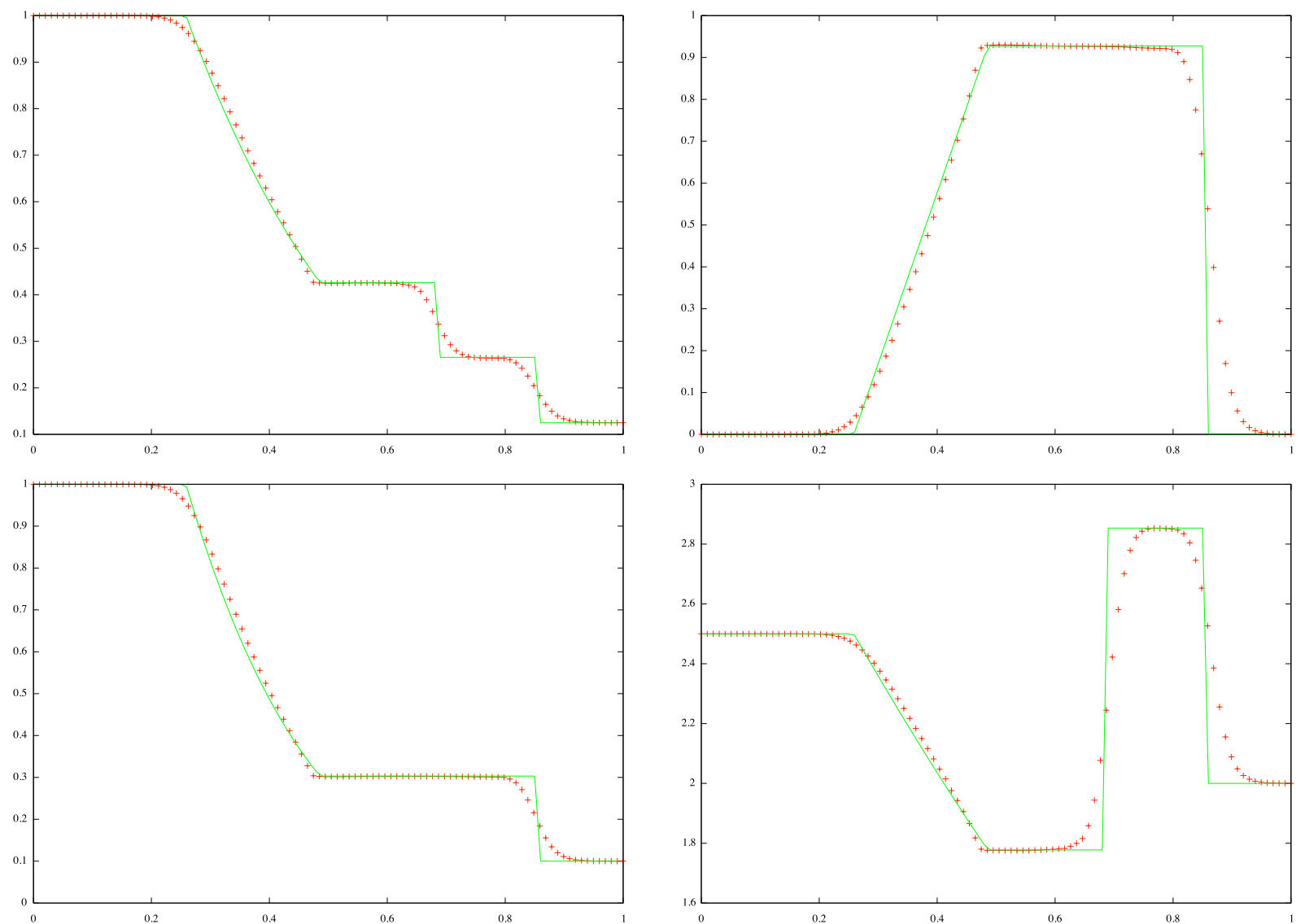

FIGURE 3. Sod shock tube, solution obtained with 100 particles compared to exact solution (solid line), from left to right and from top to bottom: density, velocity, pressure, thermal energy.

Because we have assumed that $T V\left(u_{0}\right)<+\infty$, we have also on the discrete level

$$
\sum_{j}\left|u_{j+1}^{0}-u_{j}^{0}\right|<+\infty
$$

We define the piecewise constant function

$$
u^{\Delta x}(x, t)=u_{j}^{n} \text {, for }(x, t) \in\left[x_{j+1 / 2}, x_{j-1 / 2}\right) \times\left[t_{n}, t_{n+1}\right) .
$$

The velocity of the particles is evaluated with second-order accuracy, in order that a particle scheme with a second-order kernel is really second-order. We consider the TVD particle scheme built with the kernels $\Lambda_{2}$ and $\bar{M}_{3}$ as presented in Section 4. If we name $G_{j+1 / 2}^{2}$ the flux of the $\Lambda_{2}$ kernel, and $\bar{G}^{3}{ }_{j+1 / 2}$ the flux of the $\bar{M}_{3}$ kernel, the TVD particle scheme can be expressed as

$$
u_{j}^{n+1}=u_{j}-\lambda\left[\left(1-\phi_{j+1 / 2}\right) G_{j+1 / 2}^{2}+\phi_{j+1 / 2} \bar{G}^{3}{ }_{j+1 / 2}-\left(1-\phi_{j-1 / 2}\right) G_{j-1 / 2}^{2}-\phi_{j-1 / 2} \bar{G}^{3}{ }_{j-1 / 2}\right] .
$$


With the expression of the flux obtained in Section 3, this can be re-written as

$$
\begin{aligned}
u_{j}^{n+1}= & u_{j}-\sum_{k=1}^{d} \sum_{i=0}^{N} \frac{\lambda^{i}}{i !}\left[\left(1-\phi_{j+1 / 2}\right)\left(-\Lambda_{2}^{(i)}(k) \sum_{a=1}^{k} u_{j+a} \tilde{g}_{j+a}^{i}+\Lambda_{2}^{(i)}(-k) \sum_{a=-k+1}^{0} u_{j+a} \tilde{g}_{j+a}^{i}\right)\right. \\
& +\phi_{j+1 / 2}\left(-\bar{M}_{3}^{(i)}(k) \sum_{a=1}^{k} u_{j+a} \tilde{g}_{j+a}^{i}+\bar{M}_{3}^{(i)}(-k) \sum_{a=-k+1}^{0} u_{j+a} \tilde{g}_{j+a}^{i}\right) \\
& -\left(1-\phi_{j-1 / 2}\right)\left(-\Lambda_{2}^{(i)}(k) \sum_{a=0}^{k-1} u_{j+a} \tilde{g}_{j+a}^{i}+\Lambda_{2}^{(i)}(-k) \sum_{a=-k}^{-1} u_{j+a} \tilde{g}_{j+a}^{i}\right) \\
& \left.-\phi_{j-1 / 2}\left(-\bar{M}_{3}^{(i)}(k) \sum_{a=0}^{k-1} u_{j+a} \tilde{g}_{j+a}^{i}+\bar{M}_{3}^{(i)}(-k) \sum_{a=-k}^{-1} u_{j+a} \tilde{g}_{j+a}^{i}\right)\right] .
\end{aligned}
$$

Therefore we can write for the expression of such a TVD particle scheme

$$
u_{j}^{n+1}=\sum_{k=-d}^{d} u_{j+k}^{n} \bar{\Lambda}\left(k, \phi_{j-1 / 2}, \phi_{j+1 / 2}, \lambda \tilde{g}_{j+k}\right) .
$$

The remeshing weights depend on the values of the other neighbouring particles, through the limiting function $\phi$. The two interpolation kernels used in this nonlinear combination can in fact be any interpolation kernel preserving the first two momenta and giving TVD remeshing formulas, and any other more accurate interpolation kernel, as long as the combination of their fluxes is TVD and consistent.

Proposition 5.1. The particle scheme defined by (5.1), built as a TVD combination of the kernels $\Lambda_{2}$ and $\bar{M}_{3}$ as described in 4 , and satisfying the CFL condition

$$
\lambda\left|\tilde{g}\left(u_{k}^{n}\right)\right|<1 / 2 \text { and } \lambda\left|g\left(u_{k}^{n}\right)\right|<1 / 2 \text { for all }(k, n)
$$

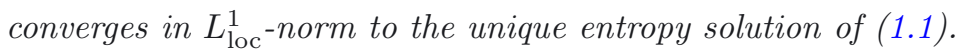

Proof. We first need to prove that the TVD particle scheme is stable in $L^{\infty}\left(\mathbb{R}^{+} \times \mathbb{R}\right)$ norm. In that purpose we assume that the particle scheme is not stable in this norm and show that it leads to a contradiction. Without loss of generality one can assume for example that, for all $M>0$, there exist $n_{0}, j_{0}$ such that $u_{j_{0}}^{n_{0}}>M$. Because the particle scheme can be written in conservative form, we have

$$
\Delta x \sum_{j} u_{j}^{n+1}=\Delta x \sum_{k} u_{k}^{0} .
$$

There exists necessarily an index $j_{1}$ such that $u_{j_{1}}^{n_{0}}<\frac{M}{2}$. Thus,

$$
\sum_{j}\left|u_{j+1}^{n_{0}}-u_{j}^{n_{0}}\right| \geq\left|u_{j_{0}}^{n_{0}}-u_{j_{1}}^{n_{0}}\right| \geq \frac{M}{2},
$$

which is not possible if we choose $M$ such that $\frac{M}{2}>\sum_{j}\left|u_{j+1}^{0}-u_{j}^{0}\right|$. Therefore the TVD particle scheme in bounded in $L^{\infty}\left(\mathbb{R}^{+} \times \mathbb{R}\right)$ norm, and thus also bounded in $L^{1}(\Omega)$ for every bounded open set $\Omega \in \mathbb{R}^{+} \times \mathbb{R}$.

Now we want to prove that for every bounded open set $\Omega \in \mathbb{R}^{+} \times \mathbb{R}$ the total variation of the scheme over $\Omega$ is bounded. Cottet and Magni have devised their flux limited particle method in order to have

$$
\sum_{j}\left|u_{j+1}^{n+1}-u_{j}^{n+1}\right| \leq \sum_{j}\left|u_{j+1}^{n}-u_{j}^{n}\right| .
$$


We use a result in [19], saying that if a conservative scheme with a Lipschtiz-continuous flux satisfies the property $\sum_{k}\left|u_{j+1}^{n+1}-u_{j}^{n+1}\right| \leq \sum_{k}\left|u_{j+1}^{n}-u_{j}^{n}\right|$, then the total variation of the scheme is bounded. The flux $G_{j+1 / 2}^{1}+$ $\phi_{j+1 / 2}\left(G_{j+1 / 2}^{2}-G_{j+1 / 2}^{1}\right)$ with $G_{j+1 / 2}^{1}$ and $G_{j+1 / 2}^{2}$ defined as in Section 3.2 is locally Lipschitz-continuous, and the scheme is stable in $L^{\infty}\left(\mathbb{R}^{+} \times \mathbb{R}\right)$ norm. Thus the total variation of the scheme is bounded.

Now we follow the type of reasoning presented in [19]: we consider a sequence of numerical approximations $u^{\left(\Delta x_{i}\right)}$ obtained with the particle scheme with grid parameters $\Delta x_{i}$ tending to zero when $i$ tends to infinity. We assume that this sequence does not converge in $L^{1}(\Omega)$ toward $u$ the entropy solution of (1.1), and show that this assumption leads to a contradiction. If the particle scheme does not converge toward $u$, then there exists some $\epsilon>0$ and a subsequence $u^{\left(\tilde{\left.\Delta x_{i}\right)}\right.}$ such that:

$$
\left\|u^{\left(\tilde{\left.\Delta x_{i}\right)}\right.}-u\right\|_{L^{1}(\Omega)}>\epsilon \text { for all } i .
$$

Because $u^{\left(\tilde{\left.\Delta x_{i}\right)}\right.}$ is bounded in $L^{1}(\Omega) \cap T V(\Omega)$, and because of the Helly theorem, one can extract a subsubsequence $u^{\left(\Delta \bar{x}_{i}\right)}$ that converges in $L^{1}(\Omega)$. Let us call $\bar{u}$ the limit of $u^{\left(\Delta x_{i}\right)}$. The particle scheme is bounded in $L^{\infty}\left(\mathbb{R}^{+} \times \mathbb{R}\right)$ norm, can be written in conservative form consistent with equation (1.1), thus because of the Lax-Wendroff theorem, $\bar{u}$ is necessarily a weak solution of (1.1).

Now we want to prove that $\bar{u}$ satisfies a weak entropy inequality for all Kruzkov entropies, i.e., for all $\phi \in C_{0}^{1}\left(\mathbb{R}^{+} \times \mathbb{R}\right), \phi \geq 0$, for all $K \in \mathbb{R}$,

$$
\int_{0}^{+\infty} \int_{-\infty}^{+\infty}\left\{\partial_{t} \phi|\bar{u}-K|+\operatorname{sgn}(\bar{u}-K)(g(\bar{u}) \bar{u}-g(K) K) \partial_{x} \phi\right\} \mathrm{d} x \mathrm{~d} t+\int_{-\infty}^{+\infty}|\bar{u}(0, x)-K| \partial_{x} \phi(0, x) \mathrm{d} x \geq 0 .
$$

This will be possible thanks to the form of the particle scheme: the new particle values are expressed as a weighted sum of the old values, unlike finite-difference schemes like the Lax-Wendroff scheme. We define

$$
\begin{aligned}
(I)=\sum_{n=0}^{\infty} & \sum_{j} \frac{1}{\Delta t}\left[\phi_{j}^{n}\left|u_{j}^{n+1}-K\right|-\phi_{j}^{n} \sum_{k} \operatorname{sgn}\left(u_{k}^{n}-K\right)\left[u_{k}^{n} \bar{\Lambda}\left(k-j, \phi_{j-1 / 2}, \phi_{j+1 / 2}, \lambda \tilde{g}_{k}^{n}\right)\right.\right. \\
& \left.\left.-K \bar{\Lambda}\left(k-j, \phi_{j-1 / 2}, \phi_{j+1 / 2}, \lambda \tilde{g}(K)\right)\right]\right] \Delta x \Delta t
\end{aligned}
$$

with $\phi \in C_{0}^{1}\left(\mathbb{R}^{+} \times \mathbb{R}\right)$ a positive function whose support is included in $[-D, D] \times[0, T]$. We firstly want to prove that

$$
\lim _{\Delta t \rightarrow 0}(I)=-\int_{0}^{+\infty} \int_{-\infty}^{+\infty}\left\{\partial_{t} \phi|\bar{u}-K|+\operatorname{sgn}(\bar{u}-K)(g(\bar{u}) \bar{u}-g(K) K) \partial_{x} \phi\right\} \mathrm{d} x \mathrm{~d} t-\int_{-\infty}^{+\infty}|\bar{u}(0, x)-K| \partial_{x} \phi(0, x) \mathrm{d} x
$$

and secondly that

$$
\lim _{\Delta t \rightarrow 0}(I) \leq 0
$$

By exchanging the indices $j$ and $k$ and making a change of variable on $k$ we get

$$
\begin{aligned}
(I)= & \sum_{n=0}^{\infty} \sum_{j} \frac{1}{\Delta t}\left[\phi_{j}^{n}\left|u_{j}^{n+1}-K\right|-\operatorname{sgn}\left(u_{j}^{n}-K\right) u_{j}^{n} \sum_{k} \phi_{j-k}^{n} \bar{\Lambda}\left(k, \phi_{j-k-1 / 2}, \phi_{j-k+1 / 2}, \lambda \tilde{g}_{j}^{n}\right)\right. \\
& \left.+\operatorname{sgn}\left(u_{j}^{n}-K\right) K \sum_{k} \phi_{j-k}^{n} \bar{\Lambda}\left(k, \phi_{j-k-1 / 2}, \phi_{j-k+1 / 2}, \lambda \tilde{g}(K)\right)\right] \Delta x \Delta t .
\end{aligned}
$$


With a change of variables for indices $n$, the equation becomes

$$
\begin{aligned}
(I)= & \sum_{n=1}^{\infty} \sum_{j} \frac{1}{\Delta t}\left[\phi_{j}^{n-1}\left|u_{j}^{n}-K\right|-\operatorname{sgn}\left(u_{j}^{n}-K\right) u_{j}^{n} \sum_{k} \phi_{j-k}^{n} \bar{\Lambda}\left(k, \phi_{j-k-1 / 2}, \phi_{j-k+1 / 2}, \lambda \tilde{g}_{j}^{n}\right)\right. \\
& \left.+\operatorname{sgn}\left(u_{j}^{n}-K\right) K \sum_{k} \phi_{j-k}^{n} \bar{\Lambda}\left(k, \phi_{j-k-1 / 2}, \phi_{j-k+1 / 2}, \lambda \tilde{g}(K)\right)\right] \Delta x \Delta t \\
& -\frac{1}{\Delta t} \sum_{j}\left[\operatorname{sgn}\left(u_{j}^{0}-K\right) u_{j}^{0} \sum_{k} \phi_{j-k}^{0} \bar{\Lambda}\left(k, \phi_{j-k-1 / 2}, \phi_{j-k+1 / 2}, \lambda \tilde{g}_{j}^{0}\right)\right. \\
& \left.-\operatorname{sgn}\left(u_{j}^{0}-K\right) K \sum_{k} \phi_{j-k}^{0} \bar{\Lambda}\left(k, \phi_{j-k-1 / 2}, \phi_{j-k+1 / 2}, \lambda \tilde{g}(K)\right)\right] \Delta x \Delta t .
\end{aligned}
$$

The idea is to recognize in the terms $\sum_{k} \phi_{j-k}^{n} \bar{\Lambda}\left(k, \phi_{j-k-1 / 2}, \phi_{j-k+1 / 2}, \lambda \tilde{g}_{j}^{n}\right)$ a discrete approximation of $\phi\left(x_{j}, t^{n}\right)-\Delta t g\left(u_{j}^{n}\right) \partial_{x} \phi\left(x_{j}, \Delta t\right)$. The TVD remeshing scheme is obtained by combining the fluxes of a linear interpolation kernel, which gives a first-order scheme, and another kernel which provides at least second-order interpolation. The resulting scheme is thus at least first-order accurate on every grid point. Therefore, we can write as in the Proof of Proposition 3.4 in Section 3

$$
\begin{gathered}
\sum_{k} \phi_{j-k}^{n} \bar{\Lambda}\left(k, \phi_{j-k-1 / 2}, \phi_{j-k+1 / 2}, \lambda \tilde{g}_{j}^{n}\right)=\phi\left(x_{j}, t^{n}\right)-\Delta t g\left(u_{j}^{n}\right) \partial_{x} \phi\left(x_{j}, t^{n}\right)+O\left(\Delta t^{2}\right) \\
\sum_{k} \phi_{j-k}^{n} \bar{\Lambda}\left(k, \phi_{j-k-1 / 2}, \phi_{j-k+1 / 2}, \lambda \tilde{g}(K)\right)=\phi\left(x_{j}, t^{n}\right)-\Delta t g(K) \partial_{x} \phi\left(x_{j}, t^{n}\right)+O\left(\Delta t^{2}\right) .
\end{gathered}
$$

Therefore when $\Delta t$ tends to zero

$$
(I) \rightarrow-\int_{0}^{+\infty} \int_{-\infty}^{+\infty} \partial_{t} \phi|u-K|+\operatorname{sgn}(u-K)(g(u) u-g(K) K) \partial_{x} \phi \mathrm{d} x \mathrm{~d} t-\int_{-\infty}^{+\infty}|u(0, x)-K| \partial_{x} \phi(0, x) \mathrm{d} x .
$$

Now we want to prove that $\lim _{\Delta t \rightarrow 0}(I) \leq 0$. By noting $\Phi$ the maximum of $|\phi|$ on $[-D, D] \times[0, T]$ we have

$$
\begin{aligned}
(I) \leq & \Phi \sum_{n=0}^{T / \Delta t} \Delta t \sum_{j, j \Delta x \in[-D, D]}|| u_{j}^{n+1}-K \mid-\sum_{k} \operatorname{sgn}\left(u_{k}^{n}-K\right)\left[u_{k}^{n} \bar{\Lambda}\left(k-j, \phi_{j-1 / 2}, \phi_{j+1 / 2}, \lambda \tilde{g}_{k}^{n}\right)\right. \\
& \left.-K \bar{\Lambda}\left(k-j, \phi_{j-1 / 2}, \phi_{j+1 / 2}, \lambda \tilde{g}(K)\right)\right] \mid \Delta x .
\end{aligned}
$$

We define

$$
\begin{aligned}
\left(B^{n}\right)= & \sum_{j, j \Delta x \in[-D, D]}|| u_{j}^{n+1}-K \mid-\sum_{k} \operatorname{sgn}\left(u_{k}^{n}-K\right)\left[u_{k}^{n} \bar{\Lambda}\left(k-j, \phi_{j-1 / 2}, \phi_{j+1 / 2}, \lambda \tilde{g}_{k}^{n}\right)\right. \\
& \left.-K \bar{\Lambda}\left(k-j, \phi_{j-1 / 2}, \phi_{j+1 / 2}, \lambda \tilde{g}(K)\right)\right] \mid \Delta x .
\end{aligned}
$$

We want to prove that $\left(B^{n}\right)$ tends to zero when $\Delta x$ tends to zero. Because of the definition of the particle scheme (5.1)

$$
\begin{aligned}
\left(B^{n}\right)= & \sum_{j, j \Delta x \in[-D, D]}|| \sum_{k} u_{k}^{n} \bar{\Lambda}\left(k-j, \phi_{j-1 / 2}, \phi_{j+1 / 2}, \lambda \tilde{g}_{k}^{n}\right)-K \mid \\
& -\sum_{k} \operatorname{sgn}\left(u_{k}^{n}-K\right)\left[u_{k}^{n} \bar{\Lambda}\left(k-j, \phi_{j-1 / 2}, \phi_{j+1 / 2}, \lambda \tilde{g}_{k}^{n}\right)-K \bar{\Lambda}\left(k-j, \phi_{j-1 / 2}, \phi_{j+1 / 2}, \lambda \tilde{g}(K)\right)\right] \mid \Delta x .
\end{aligned}
$$


The kernels involved in the formula of $\Lambda$ have a compact support, and we have assumed that a CFL condition was satisfied. So there exists a real $S$ such that

$$
\bar{\Lambda}\left(k-j, \phi_{j-1 / 2}, \phi_{j+1 / 2}, \lambda \tilde{g}_{k}\right)=0 \text { if } k-j>S .
$$

Let $\eta>0$ be a real such that $S \Delta x<\eta \forall \Delta x$. Because $u$ is locally bounded, for all $\epsilon>0$ there exists $u_{\epsilon} \in C^{1}([-D-\eta, D+\eta] \times[0, T])$ such that

$$
\left\|u(t, .)-u_{\epsilon}(t, .)\right\|_{L^{1}([-D-\eta, D+\eta])} \leq \epsilon .
$$

We split $\left(B^{n}\right)$ into several terms.

$$
\begin{aligned}
\left(B^{n}\right) \leq & \underbrace{\sum_{j, j \Delta x \in[-D, D]}\left|\sum_{k}\left(u_{k}^{n}-u_{\epsilon}\left(x_{k}, t^{n}\right)\right) \bar{\Lambda}\left(k-j, \phi_{j-1 / 2}, \phi_{j+1 / 2}, \lambda \tilde{g}_{k}^{n}\right)\right| \Delta x}_{(a)} \\
& +\underbrace{\sum_{j, j \Delta x \in[-D, D]}\left|\sum_{k} u_{\epsilon}\left(x_{k}, t^{n}\right) \bar{\Lambda}\left(k-j, \phi_{j-1 / 2}, \phi_{j+1 / 2}, \lambda \tilde{g}_{k}^{n}\right)-u_{\epsilon}\left(x_{j}, t^{n}\right)\right| \Delta x}_{(b)} \\
& +\underbrace{\sum_{j, j \Delta x \in[-D, D]} \mid \operatorname{sgn}\left(u_{\epsilon}\left(x_{j}, t^{n}\right)-K\right)\left(u_{\epsilon}\left(x_{j}, t^{n}\right)-K\right) \Delta x}_{(c)} \\
& \underbrace{\sum_{k} \operatorname{sgn}\left(u_{\epsilon}\left(x_{k}, t^{n}\right)-K\right)\left(u_{\epsilon}\left(x_{k}, t^{n}\right) \bar{\Lambda}\left(k-j, \phi_{j-1 / 2}, \phi_{j+1 / 2}, \lambda \tilde{g}_{k}^{n}\right)\right.}_{(c)} \\
& \underbrace{\left.-K \bar{\Lambda}\left(k-j, \phi_{j-1 / 2}, \phi_{j+1 / 2}, \lambda \tilde{g}(K)\right)\right) \mid}_{(c)} \underbrace{}_{(d)} \underbrace{\underbrace{}_{j, j \Delta x \in[-D, D]} \mid \underbrace{}_{k}\left(u_{\epsilon}\left(x_{k}, t^{n}\right)-K\right)\left[u_{\epsilon}\left(x_{k}, t^{n}\right) \bar{\Lambda}\left(k-j, \phi_{j-1 / 2}, \phi_{j+1 / 2}, \lambda \tilde{g}_{k}^{n}\right)\right.}_{\sum_{(d)} \operatorname{sgn}\left(u_{k}^{n}-K\right)\left[u_{k}^{n} \bar{\Lambda}\left(k-j, \phi_{j-1 / 2}, \phi_{j+1 / 2}, \lambda \tilde{g}_{k}^{n}\right)-K \bar{\Lambda}\left(k-j, \phi_{j-1 / 2}, \phi_{j+1 / 2}, \lambda \tilde{g}(K)\right)\right] \mid} \\
& \underbrace{}_{\left.K \bar{\Lambda}\left(k-j, \phi_{j-1 / 2}, \phi_{j+1 / 2}, \lambda \tilde{g}(K)\right)\right]}
\end{aligned}
$$


The first term can be bounded as

$$
\begin{aligned}
(a) & \leq \sum_{j, j \Delta x \in[-D, D]} \sum_{k}\left|u_{k}^{n}-u_{\epsilon}\left(x_{k}, t^{n}\right)\right|\left|\bar{\Lambda}\left(k-j, \phi_{j-1 / 2}, \phi_{j+1 / 2}, \lambda \tilde{g}_{k}^{n}\right)\right| \Delta x \\
& \leq \sum_{k, k \Delta x \in[-D-S \Delta x, D+S \Delta x]}\left|u_{k}^{n}-u_{\epsilon}\left(x_{k}, t^{n}\right)\right| \sum_{j,|j-k| \leq S}\left|\bar{\Lambda}\left(k-j, \phi_{j-1 / 2}, \phi_{j+1 / 2}, \lambda \tilde{g}_{k}^{n}\right)\right| \Delta x \\
& \leq 2 S|| \bar{\Lambda} \|_{\infty} \sum_{k, k \Delta x \in[-D-S \Delta x, D+S \Delta x]}\left|u_{k}^{n}-u_{\epsilon}\left(x_{k}, t^{n}\right)\right| \Delta x \\
& \leq 2 S|| \bar{\Lambda}||_{\infty} \int_{-D-(S+1 / 2) \Delta x}^{D+(S+1 / 2) \Delta x}\left|u^{\Delta x}(x, t)-u_{\epsilon}(x, t)\right| \mathrm{d} x \\
& +2 S|| \bar{\Lambda} \|_{\infty} \sum_{k, k \Delta x \in[-D-S \Delta x, D+S \Delta x]}\left|\int_{(k-1 / 2) \Delta x}^{(k+1 / 2) \Delta x} u_{\epsilon}(x, t)-u_{\epsilon}\left(x_{k}, t^{n}\right) \mathrm{d} x\right| .
\end{aligned}
$$

Thus (a) tends to zero when $\Delta x$ tend to zero. One can prove similarly that $(d)$ tends to zero. The term $(b)$ can be rewritten

$$
\begin{aligned}
(b)= & \left.\sum_{j, j \Delta x \in[-D, D]} \mid \sum_{k} u_{\epsilon}\left(x_{k}, t^{n}\right) \bar{\Lambda}\left(k-j, \phi_{j-1 / 2}, \phi_{j+1 / 2}, \lambda \tilde{g}_{k}^{n}\right)-\sum_{k} u_{\epsilon}\left(x_{j}, t^{n}\right)\right) \Lambda_{j, k}\left(k-j+\lambda \tilde{g}_{k}\right) \\
& +\sum_{k} u_{\epsilon}\left(x_{j}, t^{n}\right) \bar{\Lambda}\left(k-j, \phi_{j-1 / 2}, \phi_{j+1 / 2}, \lambda \tilde{g}_{k}^{n}\right)-u_{\epsilon}\left(x_{j}, t^{n}\right) \underbrace{\sum_{k} \bar{\Lambda}\left(k-j, \phi_{j-1 / 2}, \phi_{j+1 / 2}, \lambda \tilde{g}_{j}^{n}\right)}_{=1} \mid \Delta x .
\end{aligned}
$$

The term $\sum_{k} \bar{\Lambda}\left(k-j, \phi_{j-1 / 2}, \phi_{j+1 / 2}, \lambda \tilde{g}_{j}^{n}\right)$ is equal to 1 because if we consider the TVD remeshing formula (5.1) with $u_{k}^{n}=u$ for all $k$ then

$$
u_{j}^{n+1}=\sum_{k} u \bar{\Lambda}\left(k-j, \phi_{j-1 / 2}, \phi_{j+1 / 2}, \lambda \tilde{g}(u)\right)
$$

We have, due to the property of consistency of the fluxes: $u_{j}^{n+1}=u$. Thus,

$$
\sum_{k} \bar{\Lambda}\left(k-j, \phi_{j-1 / 2}, \phi_{j+1 / 2}, \lambda \tilde{g}(u)\right)=1 .
$$

We split (b) into two terms.

$$
\begin{aligned}
(b) \leq & \underbrace{\sum_{j, j \Delta x \in[-D, D]} \sum_{k}\left|u_{\epsilon}\left(x_{k}, t^{n}\right)-u_{\epsilon}\left(x_{j}, t^{n}\right)\right|\left|\bar{\Lambda}\left(k-j, \phi_{j-1 / 2}, \phi_{j+1 / 2}, \lambda \tilde{g}_{k}^{n}\right)\right| \Delta x}_{(1)} \\
& +\underbrace{\sum_{j, j \Delta x \in[-D, D]}\left|\sum_{k} u_{\epsilon}\left(x_{j}, t^{n}\right)\right|\left|\bar{\Lambda}\left(k-j, \phi_{j-1 / 2}, \phi_{j+1 / 2}, \lambda \tilde{g}_{k}^{n}\right)-\bar{\Lambda}\left(k-j, \phi_{j-1 / 2}, \phi_{j+1 / 2}, \lambda \tilde{g}_{j}^{n}\right)\right| \Delta x}_{(2)} .
\end{aligned}
$$

The first one can be bounded by

$$
(1) \leq \sum_{k, k \Delta x \in[-D-S \Delta x, D+S \Delta x]} \sum_{j,|j-k| \leq S}\left|u_{\epsilon}\left(x_{k}, t^{n}\right)-u_{\epsilon}\left(x_{j}, t^{n}\right)\right|\left|\bar{\Lambda}\left(k-j, \phi_{j-1 / 2}, \phi_{j+1 / 2}, \lambda \tilde{g}_{k}^{n}\right)\right| \Delta x .
$$


The function $u_{\epsilon}$ belongs to $C^{1}([-D-\eta, D+\eta] \times[0, T])$, thus there exists a real $K$ such that:

$$
\forall x, y \in[-D-\eta, D+\eta]\left|u_{\epsilon}(x, t)-u_{\epsilon}(y, t)\right| \leq K|x-y| .
$$

Consequently,

$$
\begin{aligned}
(1) & \leq\|\bar{\Lambda}\|_{\infty} \sum_{k, k \Delta x \in[-D-S \Delta x, D+S \Delta x]} \sum_{j,|j-k| \leq S} K|k-j| \Delta x^{2} \\
& \leq 2 S^{2}\|\bar{\Lambda}\|_{\infty} K \sum_{k, k \Delta x \in[-D-S \Delta x, D+S \Delta x]} \Delta x^{2} \leq 4 S^{2}\|\bar{\Lambda}\|_{\infty} K\left(\frac{D}{\Delta x}+S\right) \Delta x^{2} .
\end{aligned}
$$

Therefore (1) tends to zero when $\Delta t$ tends to zero. The second term can be bounded by

$$
\begin{aligned}
(2) \leq & \left\|u_{\epsilon}\right\|_{\infty} \sum_{j, j \Delta x \in[-D, D]} \sum_{k, k \Delta x \in[-D-S \Delta x, D+S \Delta x]} \mid \bar{\Lambda}\left(k-j, \phi_{j-1 / 2}, \phi_{j+1 / 2}, \lambda \tilde{g}_{k}^{n}\right) \\
& -\bar{\Lambda}\left(k-j, \phi_{j-1 / 2}, \phi_{j+1 / 2}, \lambda \tilde{g}_{j}^{n}\right) \mid \Delta x \\
& \leq\left\|u_{\epsilon}\right\|_{\infty} \sum_{k, k \Delta x \in[-D-S \Delta x, D+S \Delta x]} \sum_{j,|j-k| \leq S} \mid \bar{\Lambda}\left(k-j, \phi_{j-1 / 2}, \phi_{j+1 / 2}, \lambda \tilde{g}_{k}\right) \\
& -\bar{\Lambda}\left(k-j, \phi_{j-1 / 2}, \phi_{j+1 / 2}, \lambda \tilde{g}\left(u_{\epsilon}\left(x_{k}, t^{n}\right)\right)\right) \mid \Delta x \\
& +\mid \bar{\Lambda}\left(k-j, \phi_{j-1 / 2}, \phi_{j+1 / 2}, \lambda \tilde{g}\left(u_{\epsilon}\left(x_{k}, t^{n}\right)\right)\right)-\bar{\Lambda}\left(k-j, \phi_{j-1 / 2}, \phi_{j+1 / 2}, \lambda \tilde{g}\left(u_{\epsilon}\left(x_{j}, t^{n}\right)\right) \mid \Delta x\right. \\
& +\left|\bar{\Lambda}\left(k-j, \phi_{j-1 / 2}, \phi_{j+1 / 2}, \lambda \tilde{g}\left(u_{\epsilon}\left(x_{j}, t^{n}\right)\right)\right)-\bar{\Lambda}\left(k-j, \phi_{j-1 / 2}, \phi_{j+1 / 2}, \lambda \tilde{g}_{j}\right)\right| \Delta x .
\end{aligned}
$$

We have assumed that

$$
\lambda\left|\tilde{g}\left(u_{k}^{n}\right)\right|<1 / 2 \text { and } \lambda\left|g\left(u_{k}^{n}\right)\right|<1 / 2 \text { for all }(k, n) .
$$

$\bar{\Lambda}$ is of class $C^{1}$. The function $g$ is also of class $C^{1}(\mathbb{R})$. We have assumed that the scheme converges in $L_{\text {loc }}^{1}$ when $\Delta x$ and $\Delta t$ tend to zero. We conclude that (2), thus (b), tend to zero when $\Delta x$ and $\Delta t$ tend to zero. With the same kind of reasoning we could also prove that $(c)$ tends to zero.

We conclude that the limit $\bar{u}$ satisfies for all $\phi \in C_{0}^{1}\left(\mathbb{R}^{+} \times \mathbb{R}\right)$, for all $K \in \mathbb{R}$

$$
-\int_{0}^{+\infty} \int_{-\infty}^{+\infty}\left\{\partial_{t} \phi|u-K|+\operatorname{sgn}(u-K)(g(u) u-g(K) K) \partial_{x} \phi\right\} \mathrm{d} x \mathrm{~d} t-\int_{-\infty}^{+\infty}|u(0, x)-K| \partial_{x} \phi(0, x) \mathrm{d} x \leq 0 .
$$

Therefore $\bar{u}$ is the unique entropy solution of (1.1), which contradicts the initial assumption. We conclude that the TVD particle scheme (5.1) converges in $L^{1}(\Omega)$ to the unique entropy solution of (1.1).

\section{Conclusion}

We have studied the consistency and accuracy properties of remeshed particle methods in the case of a scalar one-dimensional conservation law. The accuracy of the particle scheme depends on the accuracy of the interpolation kernel used. In the linear case, if the interpolation kernel preserves the first $M$ momenta then the particle scheme is of order $M-1$. In the nonlinear case, the particle scheme is a priori only of order one, because of the first-order evaluation of the particle moving, unless a correction of the evaluation of the particle velocities is used. Cottet and Magni [6] introduced recently TVD remeshing schemes for particle-grid methods. We have extended the construction of these new TVD particle schemes to nonlinear conservation laws with a possible change of velocity sign, with application to Burgers and Euler equations. Numerical results obtained in the case of the Sod shock tube for the Euler equations have been presented. Then we have proved that with these new TVD remeshing schemes the particle schemes converge toward the entropy solution. The perspectives of this work are the application of the TVD particle schemes to systems of conservation laws, for instance more complex 2D and 3D compressible flows like hydrodynamic instabilities. 


\section{REFERENCES}

[1] B. Ben Moussa and J.P. Vila, Convergence of SPH methods for scalar nonlinear conservation laws. SIAM J. Numer. Anal. 37 (2000) 863-887.

[2] W. Benz, The Numerical Modelling of Nonlinear Stellar Pulsations, Problems and Prospects, a review, in Smooth Particle Hydrodynamics: NATO ASIS Series (1989) 269-287.

[3] C. Berthon, Contribution à l'analyse numérique des équations de Navier-Stokes compressibles à deux entropies spécifiques. Application à la turbulence compressible. Ph.D. thesis, Université Paris VI (1998).

[4] M. Coquerelle and G.-H. Cottet, A vortex level set method for the two-way coupling of an incompressible fluid with colliding rigid bodies. J. Comput. Phys. 227 (2008) 9121-9137.

[5] G.-H. Cottet and P.D. Koumoutsakos, Vortex methods. Cambridge University Press (2000).

[6] G.-H. Cottet and A. Magni, TVD remeshing schemes for particle methods. C. R. Acad. Sci. Paris, Ser. I 347 (2009) $1367-1372$.

[7] G.-H. Cottet and L. Weynans, Particle methods revisited: a class of high-order finite-difference schemes. C. R. Acad. Sci. Paris, Ser. I 343 (2006) 51-56.

[8] G.-H. Cottet, B. Michaux, S. Ossia and G. Vanderlinden, A comparison of spectral and vortex methods in three-dimensional incompressible flow. J. Comput. Phys. 175 (2002) 702-712.

[9] M.W. Evans and F.H. Harlow, The particle-in-cell method for hydrodynamics calculations. Technical Report, Los Alamos Scientific Laboratory (1956).

[10] A. Ghoniem and D. Wee, Modified interpolation kernels for treating diffusion and remeshing in vortex methods. J. Comput. Phys. 213 (2006) 239-263.

[11] R.A. Gingold and J.J. Monaghan, Smoothed particle hydrodynamics: theory and application to non-spherical stars. Mon. Not. R. Astron. Soc. 181 (1977) 375-389.

[12] F.H. Harlow, Hydrodynamic problems involving large fluid distorsion. J. Assoc. Comput. Mach. 4 (1957) 137-142.

[13] A.Harten, High resolution schemes for hyperbolic conservation laws. J. Comput. Phys. 49 (1983) 357-393,.

[14] T. Hou and P.G. Lefloch, Why non-conservative schemes converge to wrong solutions: error analysis. Math. Comput. 62 (1994) $497-530$

[15] P. Koumoutsakos and S. Hieber. A Lagrangian particle level set method. J. Comput. Phys. 210 (2005) 342-367.

[16] P. Koumoutsakos and A. Leonard, High resolution simulations of the flow around an impulsively started cylinder using vortex methods. J. Fluid Mech. 296 (1995) 1-38.

[17] N. Lanson and J.P. Vila, Convergence des méthodes particulaires renormalisées pour les systèmes de Friedrichs. $C$. R. Acad. Sci. Paris, Ser. I 349 (2005) 465-470.

[18] N. Lanson and J.P. Vila, Renormalized meshfree schemes II: convergence for scalar conservation laws. SIAM J. Numer. Anal. 46 (2008) 1935-1964.

[19] R.J. LeVeque, Finite-volume methods for hyperbolic problems. Cambridge University Press (2002).

[20] A. Magni, Méthodes particulaires avec remaillage : analyse numérique nouveaux schémas et applications pour la simulation d'équations de transport. Ph.D. thesis, Université de Grenoble. Available on: http://tel.archives-ouvertes.fr/ tel-00623128/fr/ (2011).

[21] A. Magni and G.-H. Cottet, Accurate, non-oscillatory, remeshing schemes for particle methods. J. Comput. Phys. 231 (2012) $152-172$.

[22] A. Majda and S. Osher, Numerical viscosity and the entropy condition. Commun. Pure Appl. Math. 32 (1979) $797-838$.

[23] J.J. Monaghan, Why particle methods work. SIAM J. Sci. Stat. Comput 3 (1982) 422-433.

[24] J.J. Monaghan, Extrapolating B-splines for interpolation. J. Comput. Phys. 60 (1985) 253-262.

[25] J.J. Monaghan, Smoothed particle hydrodynamics. Annu. Rev. Astron. Astrophys. 30 (1992) 543-574.

[26] P. Ploumhans, G.S. Winckelmans, J.K. Salmon, A. Leonard and M.S. Warren, Vortex methods for direct numerical simulation of three-dimensional bluff body flows: application to the sphere at Re $=300,500$, and 1000. J. Comput. Phys. 178 (2002) $427-463$.

[27] P. Poncet, Topological aspects of the three-dimensional wake behind rotary oscillating circular cylinder. J. Fluid Mech. 517 (2004) 27-53

[28] G.A. Sod, A survey of several finite-difference methods for systems of nonlinear hyperbolic conservation laws. J. Comput. Phys. 27 (1978) 1-131.

[29] L. Weynans, Méthode particulaire multi-niveaux pour la dynamique des gaz, application au calcul d'écoulements multifluides. Ph.D. thesis, Université Joseph Fourier. Available on: http://tel.archives-ouvertes.fr/tel-00121346/en/ (2006). 